\title{
A Review of Indirect Bridge Monitoring Using Passing Vehicles
}

\author{
Abdollah Malekjafarian, ${ }^{1}$ Patrick J. McGetrick, ${ }^{2}$ and Eugene J. OBrien ${ }^{1}$ \\ ${ }^{1}$ School of Civil, Structural \& Environmental Engineering, University College Dublin, Newstead, Belfield, Dublin 4, Ireland \\ ${ }^{2}$ SPACE, Queen's University Belfast, David Keir Building, Belfast BT9 5AG, UK \\ Correspondence should be addressed to Eugene J. OBrien; eugene.obrien@ucd.ie
}

Received 23 October 2014; Revised 11 December 2014; Accepted 14 December 2014

Academic Editor: Mickaël Lallart

Copyright (c) 2015 Abdollah Malekjafarian et al. This is an open access article distributed under the Creative Commons Attribution License, which permits unrestricted use, distribution, and reproduction in any medium, provided the original work is properly cited.

\begin{abstract}
Indirect bridge monitoring methods, using the responses measured from vehicles passing over bridges, are under development for about a decade. A major advantage of these methods is that they use sensors mounted on the vehicle, no sensors or data acquisition system needs to be installed on the bridge. Most of the proposed methods are based on the identification of dynamic characteristics of the bridge from responses measured on the vehicle, such as natural frequency, mode shapes, and damping. In addition, some of the methods seek to directly detect bridge damage based on the interaction between the vehicle and bridge. This paper presents a critical review of indirect methods for bridge monitoring and provides discussion and recommendations on the challenges to be overcome for successful implementation in practice.
\end{abstract}

\section{Introduction}

There is an increasing demand for improved condition monitoring of transport infrastructure all over the world. Bridges are key components of transportation infrastructure and require such monitoring. There are 66,405 structurally deficient bridges in the United States (more than 11 percent of all bridges) and most of them are more than 65 years old [1]. In Europe, the majority of bridges were built in the postwar period from 1945 to 1965 [2]. The loading conditions of these bridges have changed in recent decades due to increased freight volumes and vehicle sizes. In addition, most of these bridges are subject to gradual deterioration over time and many are now structurally deficient. Rehabilitating and extending the lives of these structures raise important maintenance and safety issues.

Traditionally, bridge maintenance has relied on visual inspection methods which are highly variable and lack resolution and can only detect damage when it is visible. Therefore, structurally deficient bridges may be left undiscovered. A number of bridge collapses have occurred due to a lack of structural capacity information and Chupanit and Phromsorn [3] have suggested that visual inspection alone may not be adequate for bridge health monitoring. In countries like Japan, which is prone to natural disasters, it is recommended that monitoring of engineering infrastructure such as bridges should be conducted continuously [4]. Bridge management systems (BMSs) with integrated bridge inspections have been developed in various countries [5]. It follows that an increase in bridge inspections to address existing structurally deficient bridges has considerable cost and practical implications for road owners and managers.

Structural health monitoring (SHM) methods rely on the automatic detection of anomalous structural behaviour. SHM using the dynamic response of structures is becoming an increasingly popular part of infrastructure maintenance and management systems. Some authors distinguish four levels for categorising SHM methods, most falling within the first three levels $[6,7]$.

(1) Establish that damage is present.

(2) Establish the location.

(3) Quantify the severity.

(4) Predict the remaining service life.

One of the most popular SHM approaches is the use of structural vibration data for nondestructive damage assessment. The underlying principle is that if damage occurs in 
a structure, it leads to changes in its physical properties, for example, a loss of stiffness, and consequently causes measurable changes in its dynamic properties. Based on which dynamic properties or damage features are considered, such damage identification methods can generally be categorised in the following four groups [8].

(i) Natural frequency-based methods;

(ii) mode shape-based methods;

(iii) curvature/strain mode shape-based methods;

(iv) other methods based on modal parameters.

All of these methods can be applied to a bridge as most of them assume beam and plate type structures. In most vibration-based bridge health monitoring techniques, large numbers of sensors are installed on the structure to monitor the dynamic properties [7]. For example, many sensors have been mounted in a case study in Southern California for bridge health monitoring over an eight-year period, which included the occurrence of three earthquakes [9]. These approaches, in which sensors are installed directly on the bridge, are referred to here as direct methods and the on-site instrumentations may be costly, time-consuming, and even dangerous, depending on the location and type of bridge. The common practice is to mount the vibration sensors at different positions on the bridge and connect them to a data acquisition system. For the case where the ongoing traffic cannot be restricted for the purpose of installation, the task of equipment mounting may be risky. In addition, the implementation of SHM is not widespread for short and medium span bridges, which form the greatest proportion of bridges in service.

The idea of an indirect approach, in which the dynamic properties of bridge structures are extracted from the dynamic response of a passing vehicle, is proposed by Yang et al. $[10,11]$. Such an approach is low cost and is aimed at reducing the need for any direct installation of equipment on the bridge itself. It involves a vehicle instrumented with sensors through which dynamic properties of the bridge such as natural frequencies are extracted. Through interaction between the bridge and vehicle, the moving vehicle can be considered as both exciter and receiver. The measured vehicle response needs to include relatively high levels of bridge dynamic response in this vehicle-bridge interaction (VBI). The feasibility of this method in practice was experimentally confirmed by Lin and Yang [12] by passing an instrumented vehicle over a highway bridge in Taiwan. In the case that only bridge frequency is required, the indirect approach shows many advantages in comparison with direct methods in terms of equipment needed, specialist personnel on-site, economy, simplicity, efficiency, and mobility.

Over the past decade, many researchers have presented new methods based on indirect bridge monitoring. Although the idea only considered the fundamental frequency of the bridge at first, it was later extended to the estimation of bridge damping and mode shapes, primarily targeting level 1 SHM. In addition, some other damage detection techniques have been proposed based on the dynamic response measured on the vehicle. This review is intended to introduce and

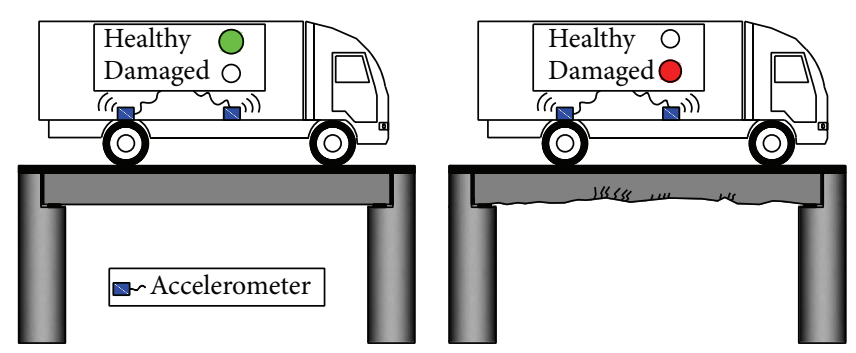

FIGURE 1: Indirect bridge health monitoring concept.

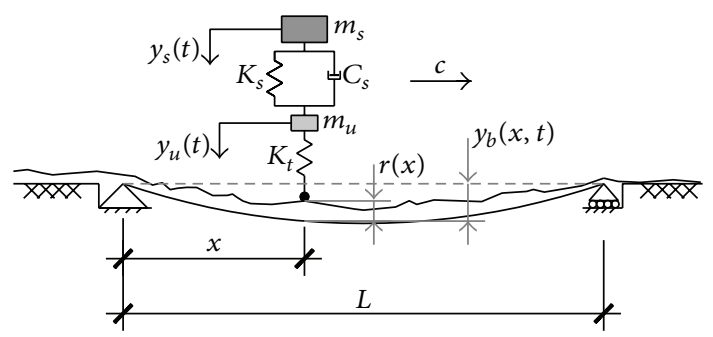

FIGURE 2: Quarter-car vehicle-bridge interaction model.

summarize these approaches and provide recommendations for future development. It also aims to guide researchers and practitioners in choosing and implementing the available bridge damage identification algorithms and signal processing methods using the dynamic response of a moving vehicle.

\section{Theoretical Background for Indirect Bridge Monitoring}

The concept of an indirect approach utilizing an instrumented vehicle, sometimes also referred to as "drive-by bridge health monitoring," is illustrated by Figure 1. The vehicle is fitted with sensors, most commonly accelerometers and most commonly on its axles. Therefore, the vehicle passing over the bridge is effectively used as a "moving sensor." Figure 2 shows an example of a simplified VBI model used for a theoretical study of such an approach, a so-called quarter-car model. This simple model can be used to briefly explain the theoretical background supporting the concept. In Figure 2, the parameters $m_{s}$ and $m_{u}$ refer to the sprung and unsprung masses, representing the vehicle body and tire assemblies, respectively. $K_{s}$ and $K_{t}$ are the suspension and tire stiffnesses, respectively, while $C_{s}$ corresponds to the suspension damping. $y_{s}$ and $y_{u}$ are the time dependent vertical displacements of the sprung and unsprung masses, respectively. The vehicle is assumed to travel at constant velocity $c$ here.

As a vehicle crosses a bridge, both vehicle and bridge vibrate and there is dynamic interaction between them. Therefore, the vehicle response is influenced by the bridge response. From the principle underlying SHM, it follows that if the bridge is damaged, for example, due to bridge bashing or concrete cracking, the stiffness, damping, and/or mass of the bridge change due to this damage and its vibration characteristics will also change. 


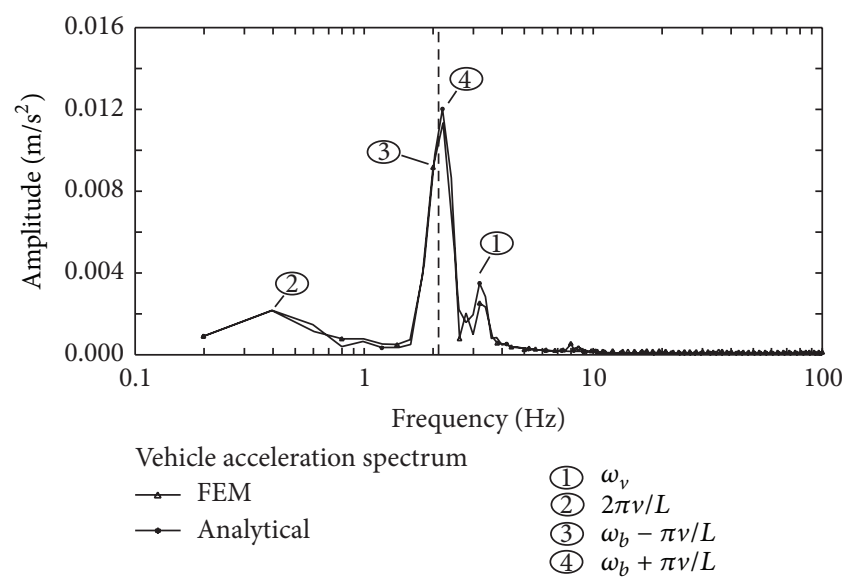

(a)

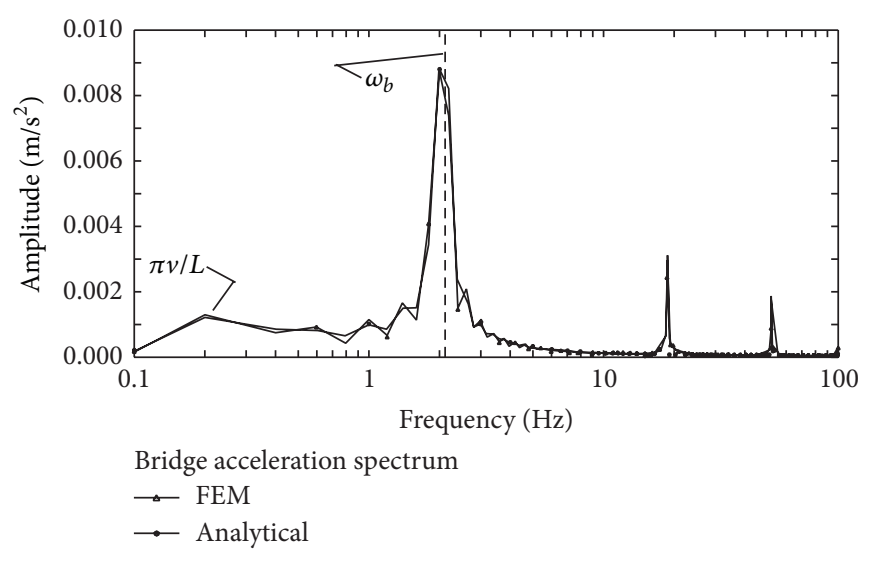

(b)

FIGURE 3: Vertical acceleration spectrum of (a) vehicle and (b) bridge midpoint. (Speed, $v$, is $10 \mathrm{~m} / \mathrm{s}, \omega_{b}$ is bridge natural frequency, and $\omega_{v}$ is vehicle frequency.) After [10].

Equation (1) gives the equations of motion for the sprung and unsprung masses:

$$
\begin{aligned}
& m_{s} \ddot{y}_{s}+C_{s}\left(\dot{y}_{s}-\dot{y}_{u}\right)+K_{s}\left(y_{s}-y_{u}\right)=0, \\
& m_{u} \ddot{y}_{u}-C_{s}\left(\dot{y}_{s}-\dot{y}_{u}\right) \\
& \quad-K_{s}\left(y_{s}-y_{u}\right)+K_{t}\left(y_{u}-y_{b}-r\right)=0,
\end{aligned}
$$

where $r$ and $y_{b}$ are the road profile displacement and bridge displacement, respectively. It can be seen from these equations that change due to damage can be detected in the vehicle response via the presence of the term $y_{b}$, the bridge displacement under the wheel of the vehicle. Therefore it is theoretically feasible to detect damage by using measurements in the vehicle alone, without using sensors on the bridge or the need to stop the vehicle. There is an added advantage of an indirect approach in that the moving sensor passes over all cross sections of the bridge, unlike sensors at fixed positions. This can provide greater spatial information compared to direct SHM $[18,19]$.

The theoretical study of the indirect monitoring concept can be extended to a range of VBI models of varying complexity incorporating, for example, vehicle pitching and rolling motions and inertial and centrifugal forces. A variety of models which allow for simplification of the problem have been used in the papers reviewed here. A comprehensive survey of the most commonly used VBI models is given by [20].

\section{Indirect Bridge Monitoring}

3.1. Indirect Bridge Frequency Monitoring. The feasibility of extracting bridge frequencies from the dynamic response of a passing vehicle is first established by Yang et al. [10]. They use a theoretical closed-form solution of vehicle-bridge interaction, the vehicle is modelled as a sprung mass while the bridge is represented by a simply supported beam, considering its first mode of vibration only. Vehicle acceleration measurements are processed using a fast Fourier transform (FFT) in order to obtain the bridge frequency. Through the analytical study, it is shown that the vehicle response is dominated by four specific frequencies: the vehicle frequency, driving frequency of the moving vehicle $(2 \pi v / L$ in Figure $3(\mathrm{a}))$, and two shifted frequencies of the bridge $\left(\omega_{b} \pm \pi v / L\right.$ in Figure 3(a)). In addition, it is shown that the extraction of bridge frequencies from the vehicle response is not restricted by any of the assumptions adopted in the analytical study. It is highlighted that frequency matching between the vehicle and bridge can be beneficial, a conclusion also reached in some subsequent studies $[20,21]$. The authors note that if the bridge and vehicle frequencies are close, vehicle resonance conditions can be achieved by adjusting the vehicle speed. As greater peak visibility is desirable, such conditions can be favourable for increasing the vehicle response magnitude and improving bridge frequency extraction. However, the speeds required to induce some resonance conditions may be unrealistic in practice unless the vehicle's dynamic characteristics can be altered. Nevertheless, it is noted that, in general, higher speeds of up to $25 \mathrm{~m} / \mathrm{s}(90 \mathrm{~km} / \mathrm{h})$ provide higher visibility of the bridge frequency, as they induce higher amplitude responses in the bridge. This is an important initial finding as it suggests that it is feasible and possibly advantageous to implement the approach at highway speeds. Yang et al. [10] find that increased bridge damping reduces visibility but do not study this effect in detail. This relationship between damping and peak magnitude has been investigated further for indirect SHM methods by a number of authors $[13,16,22$, 23]. Further investigations are carried out by Yang and Lin [11] to confirm the feasibility of the bridge frequency extraction idea.

Following publication of the concept [10], many researchers have carried out studies extending the theoretical work and targeting experimental validations. The feasibility of extracting the bridge's fundamental frequency from the response of a passing vehicle is confirmed experimentally by 
Lin and Yang [12]. The authors employ a tractor-trailer system in a field test on a $30 \mathrm{~m}$ span prestressed concrete bridge in Taiwan. The tractor acts as the bridge exciter, while the trailer acts as the receiver of bridge vibration via accelerometers. In particular, they note that lower vehicle speeds (less than $40 \mathrm{~km} / \mathrm{h}$, or $11.1 \mathrm{~m} / \mathrm{s}$ ) provide the best results due to higher spectral resolution and a lesser influence of road surface profile on the trailer response. At higher speeds, high frequency components relating to the trailer structure and road profile become more dominant in the trailer response. This observation is in contrast to the original finding [10] that higher speeds can provide better visibility. However, the influences of the road profile and to a lesser extent, the trailer system, are not considered by Yang et al. [10] which would account for this difference. These are also factors in the failure to extract higher bridge frequencies than the 1st from the trailer response. The authors recommend carrying out three tests at different speeds before confirming extraction of the bridge frequency. It is suggested that the existence of ongoing traffic is beneficial for the identification of bridge frequency from the vehicle. In particular, a heavy truck of weight 21.05 tons is used as ongoing traffic and is found to increase the bridge response, hence increasing the amplitude of the trailer response and improving frequency peak visibility.

Identifying the importance of the bridge excitation level, Oshima et al. [24] also suggest using a heavy vehicle, one which incorporates an excitation machine, in addition to the scanning vehicle, in order to yield a constant vibration on the bridge. In a field experiment, it is shown that using such an excitation machine and repeating the test several times, can be beneficial for the extraction of low-order bridge frequencies. Oshima et al. [25] also use independent component analysis (ICA) in a numerical study for the estimation the eigenfrequencies of a bridge from the vehicle response. The VBI system model is formed by combining state space and autoregressive (AR) models and a road profile is included in simulations. It is concluded that the approach estimates the road profile well but does not estimate the bridge response very well due to its dependence on the order of the assumed AR model. It is noted that several AR models should be evaluated to determine the best order while estimation was only possible at $20 \mathrm{~km} / \mathrm{h}$ and $40 \mathrm{~km} / \mathrm{h}$.

Having confirmed the feasibility of the idea, Yang and Chang [26] study the effect of several key parameters, related to vehicle speed and acceleration amplitude ratios, on the dynamic response of a vehicle passing over a bridge in order to enable a more successful extraction of the bridge frequencies from the test vehicle. The authors note that the magnitudes of shifted bridge frequency peaks in the vehicle response relative to that of the vehicle frequency peak are important for successful bridge frequency extraction. It is suggested that the most important variable is the initial vehicle/bridge acceleration amplitude ratio; the smaller this ratio, the higher the probability of successful bridge frequency extraction will be. The results of the investigation suggest that for speed, the primary consideration will be the practical amount of time needed for data collection on the bridge.

As the indirect approach proposed by Yang et al. [10] only considers the first bridge mode, Yang and Chang [27] adopt the empirical mode decomposition (EMD) technique for preprocessing of vehicle measurements in order to make the bridge frequencies of higher modes more visible. The authors show that using the proposed method, the first few frequencies of the bridge are extracted in a numerical study and the second natural frequency is detected in a full scale experimental case study. As recommended by Lin and Yang [12], at least three crossings of the bridge are completed in order to confirm successful frequency extraction. In contrast to the original study, it is suggested that it is preferable to adjust the frequencies of the test vehicle to avoid their coincidence with the bridge frequencies. In practice, this suggests using a specific monitoring vehicle for which all dynamic properties are known and/or designed.

Kim et al. [28] and Toshinami et al. [29] present the results of scaled laboratory experiments aiming at verifying the feasibility of a drive-by inspection approach incorporating frequency detection. The experimental setup consists of a two-axle moving vehicle crossing a simply supported steel beam adopted as the bridge. A scaled road surface profile is incorporated via two tracks on the bridge. The authors show that the bridge frequency is extracted from the vehicle response, although the spectra of vehicle accelerations are dominated by the vehicle frequency. Three scaled vehicle speeds investigated: $0.46 \mathrm{~m} / \mathrm{s}, 0.93 \mathrm{~m} / \mathrm{s}$ and $1.63 \mathrm{~m} / \mathrm{s}$, which correspond to speeds in reality of $10 \mathrm{~km} / \mathrm{h}, 20 \mathrm{~km} / \mathrm{h}$ and $40 \mathrm{~km} / \mathrm{h}$, respectively. Higher vehicle speeds provide larger magnitude frequency peaks in the spectra of the vehicle response; however, this also corresponds to lower spectral resolution. This suggests that speed should potentially be selected in order to provide a balance between resolution and peak magnitude, although a practical alternative may be to carry out multiple runs at different speeds $[12,27]$.

Siringoringo and Fujino [30] study a similar approach for the estimation of the bridge fundamental frequency. Theoretical simulations and a full-scale field experiment are carried out to support their approach, which is aimed at periodic bridge inspections using accelerations of a light commercial vehicle [31]. In theoretical simulations and a parametric study, it is shown that bridge frequency can be extracted from the vehicle response. In a field experiment, it is found that vehicle velocities below $30 \mathrm{~km} / \mathrm{h}$ provide the best accuracy, for which a maximum estimation error of $11.4 \%$ is obtained. Similarly to a previous study [12], it is recommended to carry out modal testing of the inspection vehicle before bridge testing. In addition, it is noted that the dynamic response of the vehicle is dominated by its own bouncing and pitching motions at the bridge entrance/exit; this is due to the bridge expansion joints. Therefore, this part of the vehicle response should not be considered when seeking to estimate bridge frequency. Due to the short amount of time a monitoring vehicle will be on the bridge, losing any portion of the signal can be significant in terms of accuracy, particularly for short span bridges. Therefore, the effect of expansion joints on the vehicle response is an important issue to be overcome. The road profile can also have a similar effect on the vehicle response to that observed by Siringoringo and Fujino [30]; that is, the vehicle frequencies will usually appear as dominant peaks in the spectrum of the vehicle response 
and this makes it difficult to detect the bridge frequency peak. Yang et al. [32] address this issue by applying some filtering techniques to remove the vehicle frequency from the spectrum. They suggest that if the vehicle natural frequencies are available, it is possible to filter them out from the spectrum and enhance the visibility of the bridge frequency.

One of the most recent attempts at extraction of bridge frequency from a passing vehicle is based on optimization. Li et al. [33] develop a new theoretical method based on the Generalized Pattern Search Algorithm (GPSA) which is a typical search method in optimization. The method is applied to the responses of a simplified vehicle-bridge interaction system consisting of a sprung mass vehicle and simply supported beam model. The algorithm is fast and an advantage of this approach is that it can identify other parameters besides the bridge's 1st natural frequency, for example, the bridge stiffness, and thus may have potential to be developed for damage detection purposes. It is shown that the bridge frequency and stiffness can be identified with reasonable accuracy. The authors show that the proposed method can still estimate the frequency accurately in the presence of noise. Although the method shows good robustness for different noise levels (with a maximum identification error of $3.3 \%$ for a signal to noise ratio of 5), the authors acknowledge that a road profile is not considered in the study, which can be one of the most significant factors in real applications.

In a theoretical investigation, Malekjafarian and OBrien [34] utilise a well-known output-only modal analysis method called frequency domain decomposition (FDD), which is based on singular value decomposition (SVD) of the power spectral density (PSD) of the measured response, to process the acceleration response from a passing vehicle. In simulations, vehicles are represented by sprung masses in a simplified VBI model. The FDD method is applied to acceleration signals measured on two following quarter-cars. The effectiveness of the FDD method for the case of close bridge and vehicle frequencies is investigated in the presence of a road profile and for a low vehicle speed of $2 \mathrm{~m} / \mathrm{s}$. The authors show that the FDD method can identify both bridge and vehicle frequencies in this case and may be a useful alternative to classical FFT analysis, which does not reveal the frequencies clearly for the simulated scenarios.

In the original work by Yang et al. [10], the vehicle acceleration spectrum for the simplified model was dominated by four frequencies: vehicle, driving, and two shifted bridge frequencies, respectively. However, in reality, variations in frequencies of the bridge and/or vehicle may occur due to the interaction between them during a vehicle crossing. Based on this, Yang et al. [35] study the variation of the instantaneous frequencies of bridges under moving vehicles. A theoretical framework is presented for the problem, considering the variation in frequencies for both the bridge and the moving vehicle. It is shown that, if a moving vehicle is to be used as a tool for measuring the bridge frequencies or for detecting bridge damage, the frequency variation caused by the moving vehicle should be taken into account, particularly for the case where the vehicle mass is not negligible compared with the bridge mass or when the resonance condition is approached.
Similar to variations observed by Yang et al. [35], Chang and Kim [36] find that the bridge frequency within a VBI system is different from that observed for the bridge system vibrating alone. In numerical simulations, a laboratory experiment, and a field experiment, they investigate the variation of bridge frequencies due to interaction with a vehicle, focusing on bridge measurements and those of a vehicle parked on the bridge. The authors derive an analytical formula to represent this variation, based on the frequency and mass ratios between the vehicle and the bridge. It is highlighted as an important consideration for VBI systems and has implications for indirect approaches as this variation, if not accounted for, may mask changes due to damage. Despite this, the authors' results indicate that the bridge frequency identified from the VBI system can also be extracted from the vehicle response.

Thus far, vehicle-based indirect approaches have been discussed. Recently, Yang et al. [37] introduce an alternative hand-drawn test "cart" (trailer) in an experimental study aiming to measure bridge frequencies in a human-controlled, efficient, and mobile way. The authors highlight that the dynamic characteristics of the test cart are crucial for the successful extraction of the frequencies of the bridge. It is mentioned that the natural frequency of the cart is the key parameter that determines the transmission of energy from the bridge to the cart. It is also recommended that the cart frequency be selected so as to be greater than the fundamental frequency of the bridge for better visibility of the bridge frequencies in the cart response. In this study, the most suitable type of wheel is selected by conducting dynamic tests on three types of wheel, namely, an inflatable wheel, a solid rubber wheel, and a PU wheel, respectively. The PU wheel consists of a metal wheel surrounded by a thin layer of polyurethane (PU). It is highlighted as the most suitable for reliable frequency extraction as it has no frequencies in the bridge frequency range of interest and it maintains better contact with the road. Heavier carts provide better bridge peak visibility as they are less sensitive to the road surface roughness, while larger ongoing traffic flows are found to be beneficial for this alternative indirect approach also. The study illustrates the feasibility of accurate extraction of bridge frequencies using a well-designed cart and, based on these results, further development of the cart is recommended. While the qualitative conclusions are based on a hand-drawn cart; they are also likely to be relevant for vehicle-based indirect approaches operating at higher speeds.

Overall, it can be concluded that, although the feasibility of extracting the bridge frequency from the response of a passing vehicle, that is, indirect bridge frequency monitoring, is now well established through theoretical and experimental investigations, there are a number of outstanding challenges that must be overcome before the approach becomes an effective and reliable system. The primary challenges that must be addressed are (1) the influence of the road profile on the vehicle response, which reduces the visibility of bridge frequency peaks and (2) the variation of the bridge frequency under a moving vehicle $[35,36]$ during VBI. As this variation may mask any frequency change caused by damage, it is 
a significant challenge for bridge damage detection using indirect monitoring of the natural frequency.

Based on the literature presented, favourable conditions have been identified for successful bridge frequency extraction in practice as follows.

(1) Speeds below $40 \mathrm{~km} / \mathrm{h}$ generally provide better results due to improved spectral resolution and reduced influence of road profile on the vehicle response.

(2) Multiple bridge crossings (at least 3) should be carried out at different vehicle speeds.

(3) Bridge excitation can be increased to improve bridge frequency peak visibility in the vehicle acceleration spectra. This can be done using a heavy vehicle, with or without an excitation machine, or by testing the bridge in the presence of other (ongoing) traffic. Increasing the vehicle speed will also have this effect but is not always recommended due to the consequent reduction in spectral resolution.

(4) Initial vehicle/bridge acceleration amplitude ratio should be small.

(5) Dynamic properties of the test vehicle or trailer should be obtained or calibrated a priori.

3.2. Indirect Identification of Bridge Damping. Damping is another dynamic property which has been found to be damage sensitive [38, 39]. A small number of studies have been carried out in recent years for the estimation of bridge damping using an instrumented vehicle.

McGetrick et al. [13] investigate the problem of identification of bridge natural frequency and damping ratio using a moving instrumented vehicle. The authors show that in the spectra of vehicle accelerations, the magnitude of PSD at both bridge and vehicle frequency peaks decreases with increased bridge damping (Figure 4). This suggests that even if a bridge frequency peak is not found, changes in bridge damping levels can be detected by analysing vehicle frequency peaks. It is suggested that changes in the magnitude of the PSD can be used as an indicator of damage in the bridge. However, the authors also note that it is difficult to detect both frequency and damping changes in the presence of a rough road profile. The results are confirmed experimentally in scaled laboratory experiments for repeated bridge crossings and three vehicle speeds $[40,41]$. The authors detect changes of the bridge damping in the vehicle spectrum, demonstrating the potential of the method for SHM.

González et al. [22] propose a novel method for the identification of damping in a bridge using a moving instrumented vehicle, intended as a preliminary bridge condition screening method. The authors develop a six-step algorithm which uses the acceleration responses measured at the two axles of a half-car model. The bridge damping is identified with reasonable accuracy using an iterative procedure in theoretical simulations, with $88 \%$ of all simulations identifying the correct damping ratio within a 10\% margin of error, and $40 \%$ of all simulations identifying the correct value within a $1 \%$ margin of error. In the parametric study, the method is found to be relatively insensitive to road profile, low levels of

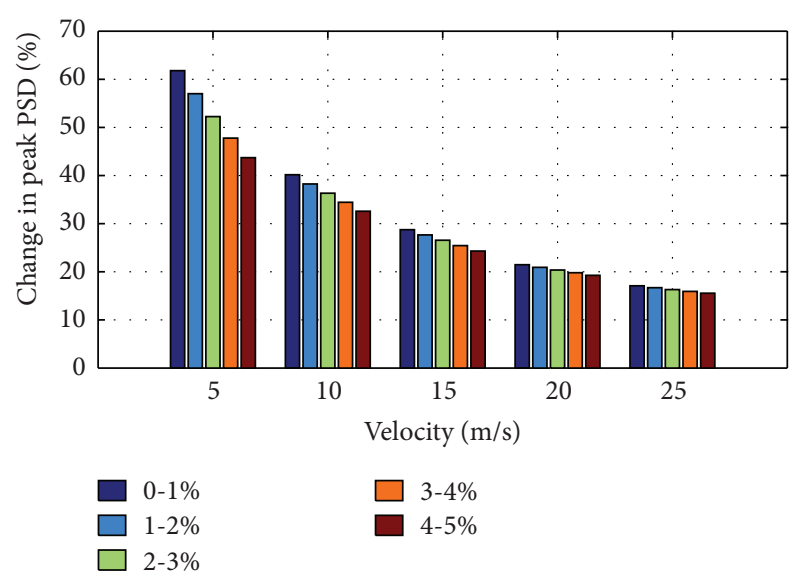

FIGURE 4: Peak vehicle PSD-bridge damping trends at bridge frequency peak for a $15 \mathrm{~m}$ span bridge for different velocities and a smooth road profile, after [13].

measurement noise and modelling errors. In particular, this method overcomes the effect of road profile highlighted in previous studies as the six-step algorithm actually estimates the road profile under each vehicle wheel; this is discussed in more detail in Section 3.4. Frequency matching between the vehicle and bridge is found to be beneficial when a pothole exists at the bridge entrance due to the resulting increase in bridge excitation. A stated advantage of this method is that it can also be extended to the estimation of the bridge stiffness. This appears to be an important consideration, as damping can be difficult to quantify in practice [42].

Although the indirect identification of bridge damping demonstrates some potential, it has certain limitations compared to approaches focusing on frequency and mode shapes, and so forth, in terms of complexity related to the aforementioned quantification of damping. Therefore, the practical performance of indirect methods focusing on damping is a significant consideration and consequently, it may be beneficial to focus on alternative bridge dynamic properties, as presented in the other sections of this paper, for example, the methodology presented by [22] for damping identification can also be applied to the identification of bridge stiffness.

3.3. Indirect Identification of Bridge Mode Shapes. As mentioned, in recent years several researchers have developed methods to identify the bridge frequency from the acceleration signal in a passing vehicle while a limited number of authors obtain the damping ratio. A number of authors have also investigated the potential of an indirect approach to identify the mode shapes of a bridge. Estimation of bridge mode shapes is very important in a bridge dynamic investigation. This is because discontinuities occur in the mode shapes at points corresponding to the locations of damage in the bridge, including slope discontinuities at points of localized damage. Mode shape curvatures may be used to find these discontinuities $[43,44]$. Furthermore, bridge mode shapes 


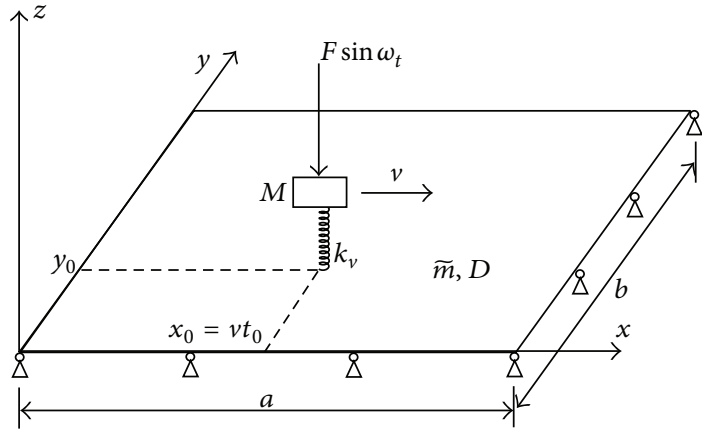

FIGURE 5: A passing tapping vehicle on a plate, after [14].

can be used as an important tool in model updating of a bridge [45].

Zhang et al. [14] model a moving vehicle passing over a bridge which is equipped with an accelerometer and shaker to control the applied force artificially, referred to as a "tapping vehicle." They present a new damage index based on the point impedance measured from the vehicle, shown in Figure 5. A controlled force is applied to the bridge from the vehicle and the response of the vehicle to the load is measured and used for constructing point impedance. It is shown that the amplitude of the spectrum obtained from the point impedance is approximately proportional to the square of the mode shape (MOSS, Figure 6) which can, in turn, be used for damage detection. Although the main purpose of the study is not estimation of bridge mode shapes, it is the first application known to the authors of an indirect technique for identification of bridge mode shape-related properties. The authors highlight that the method can be extended to obtain the absolute value of the mode shapes. It should be noted however, that the levels of accuracy obtained with this method are for relatively low vehicle speeds, less than $5 \mathrm{~m} / \mathrm{s}$ $(18 \mathrm{~km} / \mathrm{h})$, which has implications in practice.

Yang et al. [15] introduce a method for the indirect identification of bridge mode shapes based on a theoretical formulation. In the dynamic response of the test vehicle during its passage over the bridge, they show that the component response of the bridge frequency for a certain mode oscillates with a varying amplitude that is identical to the corresponding bridge mode shape. Therefore, once a bridge frequency is identified and its corresponding component response is separated from the vehicle response, the instantaneous amplitude history of the extracted component response can be regarded as being representative of the mode shape of the bridge. Hence, Yang et al. [15] propose a method based on the concept of instantaneous amplitudes obtained from the Hilbert transform of the band-pass filtered response of the vehicle. As the vehicle is effectively a moving sensor, the authors note that the indirect method can provide higher spatial resolution in mode shapes than corresponding direct approaches. Similar to Zhang et al. [14], low vehicle speeds are tested, which are 2,4 , and $8 \mathrm{~m} / \mathrm{s}(7.2,14.4$ and $28.8 \mathrm{~km} / \mathrm{h}$, resp.). It is demonstrated that the method can detect mode shapes of lower modes accurately (Figure 7) while accuracy reduces for higher vehicle speeds tested. Furthermore, additional random traffic on the bridge is found to have a negligible effect on extracted mode shape accuracy. However, the road surface profile causes a significant reduction in accuracy. The sensitivity of the method to measurement noise is not considered and the authors recommend experimental testing in order to confirm these findings.

Oshima et al. [46] investigate a theoretical bridge damage screening method which also involves the estimation of bridge mode shapes from the dynamic response of moving vehicles. In this indirect approach, the estimated mode shapes, obtained in a four-step process via singular value decomposition, are used for damage detection. The authors note that mode shapes can be more sensitive to structural damage than frequencies and damping. The vehicle configuration consists of two heavy two-axle trucks (both of $10 \mathrm{t}$ or $20 \mathrm{t}$ mass) and at least four monitoring single-axle vehicles ( $0.1 \mathrm{t}$ mass) at $1 \mathrm{~m}$ intervals, the trucks being used as bridge exciters. No additional excitation device other than the trucks is required; this is an advantage of the method over that of Zhang et al. [14], which needs a tapping vehicle system to enhance bridge vibration. Accelerations of the monitoring vehicles and the relative displacement between the axle mass and the road surface are measured; it is proposed to obtain these measurements in practice using an accelerometer and a laser distance meter, respectively, fitted to the vehicle axles. Due to ill-conditioning of the inverse problem in this method, it is found that an increase in the number of vehicles can cause a decrease of estimation accuracy in simulations. However this may be counter-balanced by the increased nodes provided by additional vehicles for mode shape construction. Four monitoring vehicles are the focus as they are found to provide higher average modal assurance criterion (MAC) values for the first 3 bridge modes than five or six vehicles. Damage scenarios include the fixing of one rotational support and a local bridge stiffness reduction of $40 \%$ at midspan. In theoretical VBI simulations considering road profiles of varying roughness, damage is detected via the proposed mode shape estimation method by analysing average MAC values. Damage is detected for vehicle speeds varying from $5 \mathrm{~m} / \mathrm{s}-15 \mathrm{~m} / \mathrm{s}(18-54 \mathrm{~km} / \mathrm{h})$, which are low compared to a highway speed range of $22.2 \mathrm{~m} / \mathrm{s}-27.8 \mathrm{~m} / \mathrm{s}(80-100 \mathrm{~km} / \mathrm{h})$ but higher than speeds proposed for other indirect methods presented in this section, which could require temporary bridge and/or lane closures. However, when measurement noise greater than $1 \%$ is considered in simulations, it is found that the damage detection approach requires an impractical number of measurements. This is a drawback for the practical application of this indirect method and the authors acknowledge the necessity for further improvement of its robustness against noise.

Malekjafarian and OBrien [47] propose the use of short time frequency domain decomposition (STFDD) for indirect identification of bridge mode shapes using responses measured from two following axles passing over a bridge. They apply the FDD method to the short time measured signals obtained at several defined stages and perform a rescaling procedure on local mode shape vectors to obtain the global mode shapes. The effect of road profile in exciting the vehicle is a significant challenge for the method. It is shown in a case 


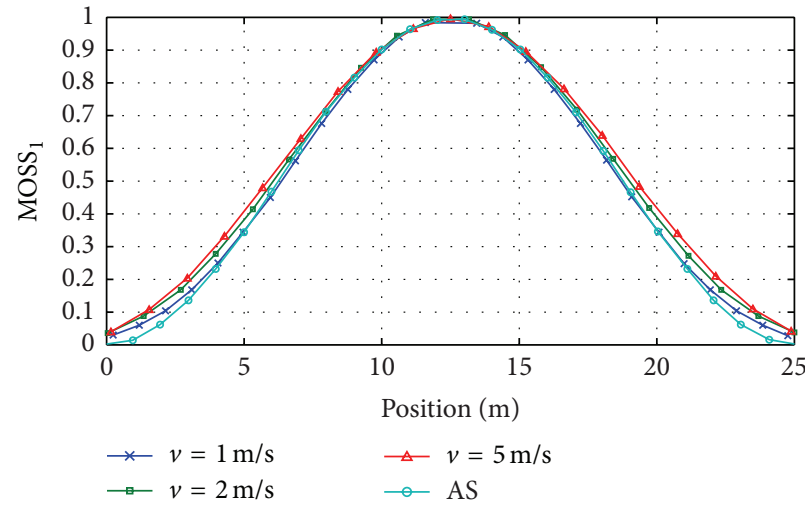

(a)

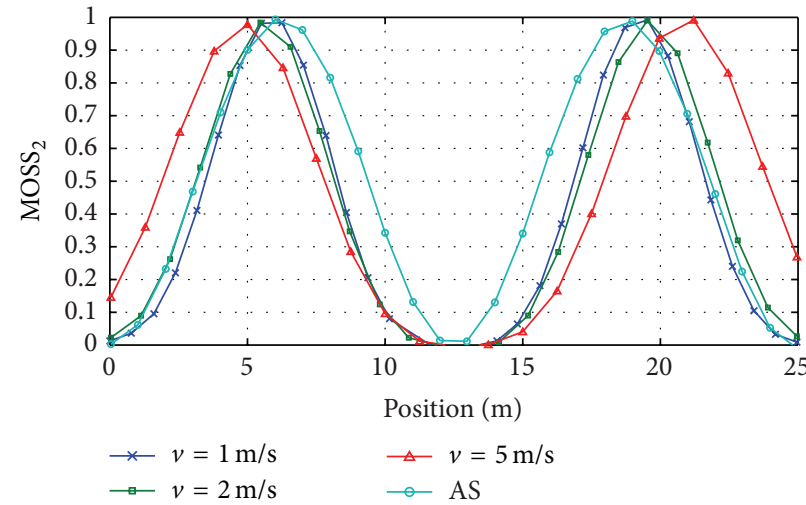

(b)

FIGURE 6: The extracted MOSS at different vehicle speeds compared with analytical solution: (a) mode 1 and (b) mode 2 ( $v$ is the velocity of the passing vehicle and AS is the analytical solution), after [14].

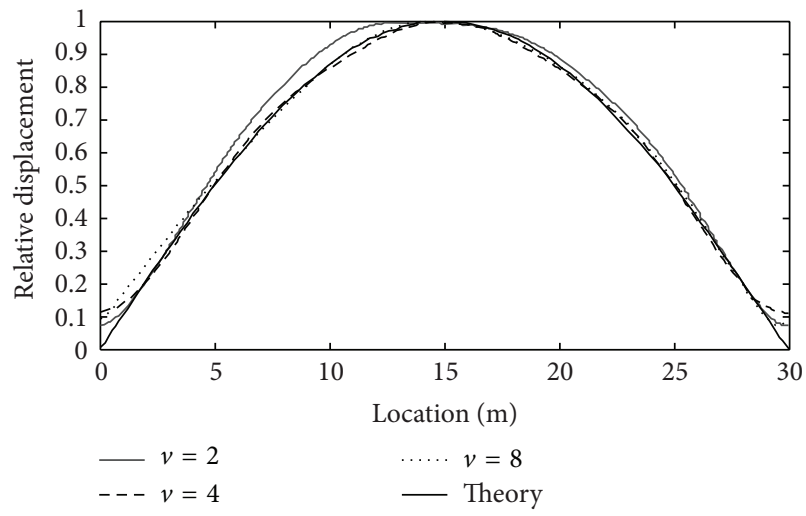

(a)

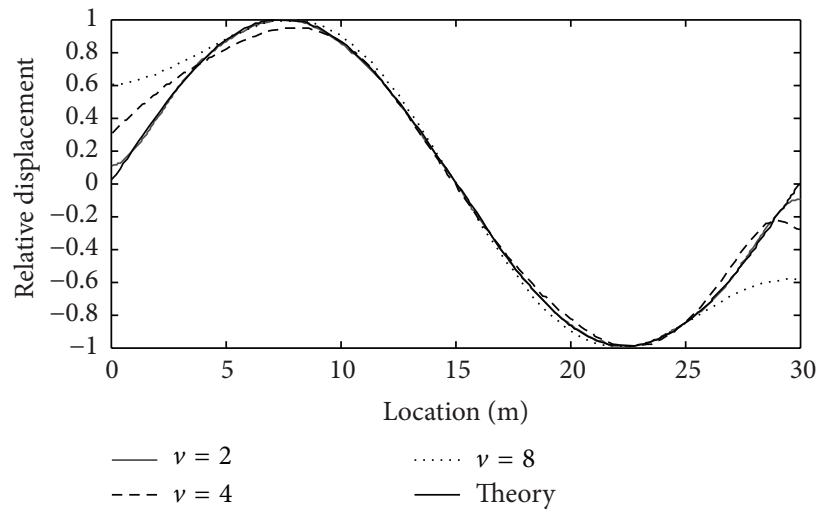

(b)

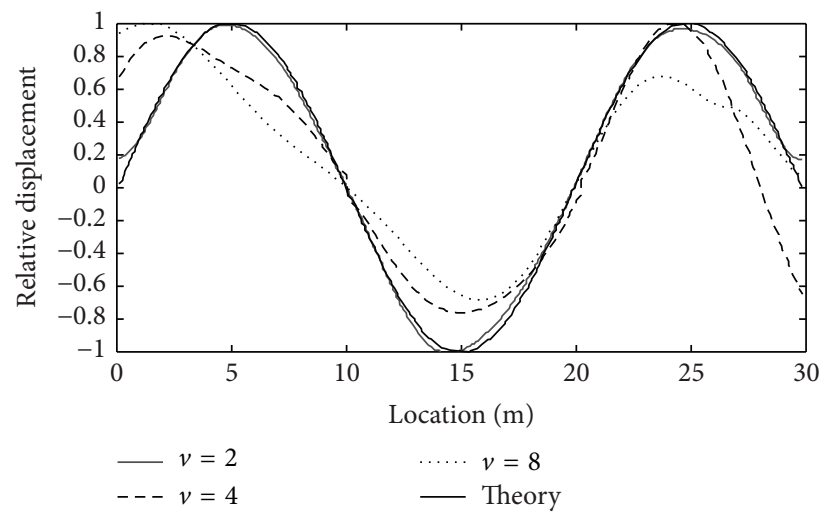

(c)

FIGURE 7: Mode shapes of the bridge obtained for different vehicle speeds ( $v, \mathrm{~m} / \mathrm{s})$ compared with theoretical result (theory): (a) 1st mode, (b) 2nd mode, and (c) 3rd mode, after [15].

study that excitation of the bridge by external forces applied to all parts of the bridge (simulating other traffic) improves the situation. In the absence of other traffic, subtraction of signals in identical axles is shown to be a feasible alternative. If noise is sufficiently low and the vehicle speed is $2 \mathrm{~m} / \mathrm{s}$ or less, mode shapes can be found with reasonable accuracy. In addition, it is found in this study that applying ongoing traffic can reduce the sensitivity of the method to noise.

Although the study by Zhang et al. [14] can be considered as the first attempt at indirect identification of bridge mode shapes, the method is based on utilizing an excitation machine via the vehicle and measuring the excitation force, 
which may be not an easy task to perform in a real case. However, a similar apparatus has been tested in a field experiment by Oshima et al. [24]. Recently, some interesting ideas $[15,46,47]$ have been proposed which are based on only the response measured of a passing vehicle. The method proposed by Yang et al. [15] provides high resolution mode shape with acceptable accuracy, particularly for the first mode shape. However, the performance of the method in the presence of measurement noise needs to be investigated. On the other hand, the methods proposed by Oshima et al. [46] and Malekjafarian and OBrien [47] both provide the bridge mode shapes with low resolution. In addition, the former study [14] shows high sensitivity to measurement noise which is an inherent characteristic of a real measurement system. Malekjafarian and OBrien [47] suggest some ideas to minimise the effect of road profile which seems to be applicable in a noisy measurement. An important consideration here is vehicle speed; these approaches all focus on very low vehicle speeds which are likely to require bridge lane closures in practice, although the approach by Oshima et al. [46] extends to a speed of $15 \mathrm{~m} / \mathrm{s}(54 \mathrm{~km} / \mathrm{h})$.

Overall, it seems that indirect bridge monitoring methods focused on the identification of bridge mode shapes have potential and possess many advantages in terms of damage detection and damage localization. However, these methods are currently limited by a lack of experimental case studies which may reveal practical challenges. Based on the existing investigations in the literature, it can be concluded that these methods need to be improved considerably and further validation is required to support successful implementation in practice, focusing on the following areas:

(1) increased mode shape resolution,

(2) reduction in sensitivity to measurement noise,

(3) implementation at higher vehicle speeds,

(4) experimental case studies.

3.4. Effect of Road Surface Profile on Vehicle-Bridge Interaction. As discussed above, the road profile on the bridge has been considered by authors to varying degrees; from ignoring it entirely to including a range of road profiles with varying roughness levels. Overall, it can be concluded that consideration of the road surface profile in indirect methods is a critical issue as it can excite vehicle-related frequencies to a much higher amplitude level than bridge-related ones, making it difficult to identify the bridge frequencies, damping ratios, and mode shapes $[48,49]$. It follows that any condition monitoring or damage detection methods which are based on these dynamic parameters are also significantly affected by the road surface profile unless some technique is applied to reduce its influence on the vehicle. Despite efforts to overcome the effect of road profile, it is still an important challenge for indirect methods that needs further investigation.

The first approach to resolving this issue is to use the excitation due to ongoing traffic to increase the relative influence of the bridge in the vehicle response [10, 26, 34, 37, 47]. Unfortunately, this is not a good assumption for short-span bridges where the probability of multiple vehicles being present simultaneously on the bridge is small.

The indirect method developed by González et al. [22] shows potential for removal of the road profile's influence. Instead of just considering the road profile as an extra input excitation to the VBI model, the algorithm identifies the damping ratio of the bridge by considering the differences in displacement under the wheels of the instrumented vehicle which effectively removes the influence of road profile. Other methods based on subtraction have also been investigated. Yang et al. [50] propose a new idea of using two connected vehicles to remove or reduce the effect of road profile from the vehicle spectrum. The authors introduce a residual spectrum by subtracting the acceleration spectra obtained from two connected axles passing over a bridge at the low speed of $2 \mathrm{~m} / \mathrm{s}$. It is demonstrated that the bridge frequency peaks are slightly improved, specifically for the case when two identical axles are used. In addition, too large of an axle spacing is not recommended, as the axles may lose their correlation.

Keenahan et al. [23] propose another version of the subtraction idea. In this study, the accelerometers are mounted on the axles of a trailer that is being towed by a truck in a truck-trailer system (Figure 8) moving at a speed of $20 \mathrm{~m} / \mathrm{s}$, much higher than that investigated by Yang et al. [50], and the difference in the accelerations between the two axles is considered. It is demonstrated that the influence of road profile is removed in the FFT spectrum of the difference between accelerations, when two identical trailer axles are used. Although the authors provide an effective method to remove the effect of road profile, the accuracy of the method is highly dependent on the similarity of the axles, as was found by Yang et al. [50] for the lower speed. In addition, it seems that the method is sensitive to environmental noise so accurate accelerometers are required.

Other indirect approaches have been developed which aim to measure road surface profile from the acceleration response of a moving vehicle $[31,51]$. The vehicle intelligent monitoring system (VIMS) presented by Fujino et al. [31] targets highway pavements and bridge expansion joints and also utilizes a GPS sensor mounted in the vehicle to identify the position where the acceleration response is recorded. The theory behind such algorithms and techniques incorporates optimisation, transfer and correlation functions which may provide a basis to further reduce the influence that road surface profile has on vehicle acceleration measurements.

\section{Damage Detection Methods Using Indirect Measurements}

This section reviews indirect methods for the detection of bridge damage that do not explicitly require the identification of bridge dynamic properties. A range of miscellaneous ideas for damage detection is reviewed in Section 4.1 including the use of moving force identification (MFI), operating deflection shape (ODS), displacement response, modal strain energy, transmissibility, and classification. Damage detection methods based on wavelet transforms are reviewed in Section 4.2 


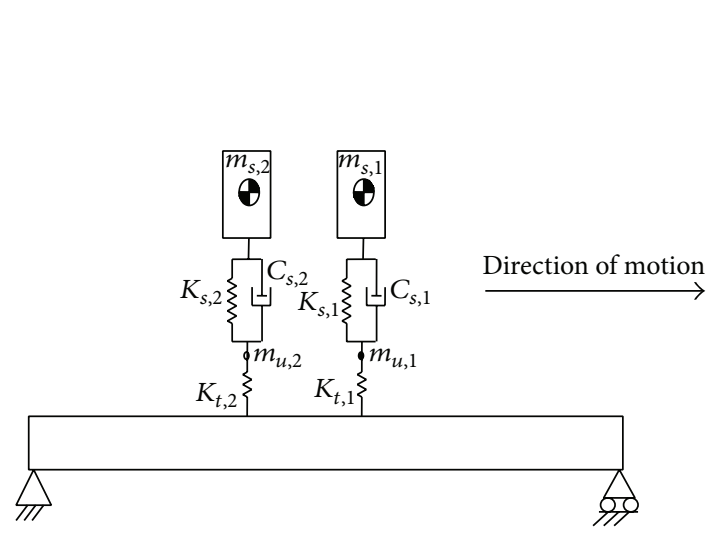

(a)

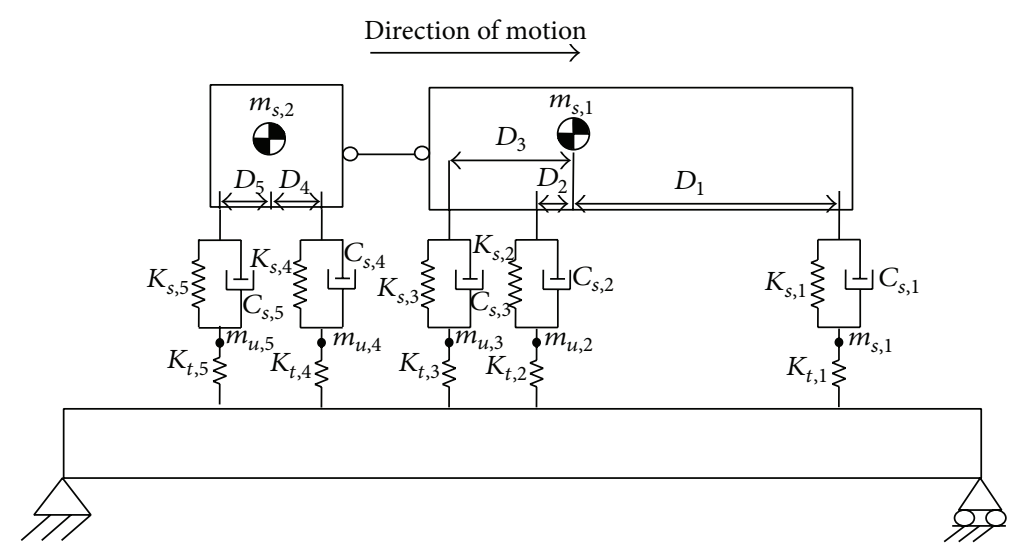

(b)

Figure 8: (a) Two identical quarter-cars, (b) truck-trailer model, after [16].

and finally methods employing a traffic speed deflectometer (TSD) are introduced in Section 4.3.

4.1. Miscellaneous Methods. As one of the earliest efforts in indirect monitoring of bridges, $\mathrm{Bu}$ et al. [52] propose a damage detection technique using an acceleration response sensitivity matrix that maps all the sensitivities as a function of vehicle position on the bridge. In a numerical investigation focusing on damage in terms of a reduction in bridge stiffness, a damage index is defined and updated in an iterative procedure. The numerical results obtained in this study show that the method is computationally stable and efficient for a quarter-car vehicle and can function in the presence of measurement noise and a road surface profile.

Kim and Kawatani [53] investigate the feasibility of bridge drive-by inspection through a laboratory experiment, focusing on damage related to bridge stiffness, similar to Bu et al. [52]. It is shown that frequency changes caused by damage can be detected by the vehicle response. Furthermore, the authors integrate the indirect method with direct bridge measurements to find the location of the damage using a damage index called ESI which defines the change in the bending rigidity of the bridge at different locations. Kim et al. [41] extend their method to include a combination of direct and indirect monitoring of short span bridges for structural diagnosis. In theoretical simulations and a scaled laboratory VBI experiment, three screening levels are presented which utilize vehicle and bridge responses both separately and together in order to identify bridge dynamic parameters and also to detect the severity and location of damage. Overall, each level is shown to be effective and for all screening levels, better identification results are found for lower vehicle speeds and for vehicles with bounce frequencies similar to the fundamental frequency of the bridge. The authors note that further study is required for accurate interpretation of damage patterns, damage sensitivity of the approaches, and finally, for the simultaneous acquisition of accurate data from the moving vehicle and the bridge.

McGetrick [54] applies Moving Force Identification, a method of finding the time history of forces applied to the bridge, and show that the calculated pattern of applied force is sensitive to bridge damage. The potential of the method to identify the global bending stiffness of the bridge is presented. Stiffness identification accuracy is found to be high for a very good road profile and low levels of signal noise, although accuracy decreases with increasing signal noise and road roughness. It is suggested that increasing the bridge displacement under the vehicle would assist with this increase.

As discussed above, Zhang et al. [14] propose a new damage index based on the point impedance measured from a tapping vehicle. It is shown that the method is very robust in the presence of noise. Although it shows very good accuracy, it is not based on the acceleration response of the vehicle only as the applied force is being controlled (by a shaker) and measured at the same time in order to construct the point impedance. Therefore, the practical application of such a moving shaker on a real bridge is an important issue to be addressed. The authors recently improved their method by using the Operating Deflection Shape Curvature (ODSC) extracted from the same device, for damage detection [55]. They use a pre-filtering process based on wavelet decomposition to obtain a smoother ODSC. Furthermore, a new damage detection algorithm called the Global Filtering Method (GFM) is proposed to eliminate the requirement of a baseline with the assumption that the intact structure is smooth and homogenous. The Gapped Smoothing Method (GSM) and GFM, based on the extracted ODSC's at relatively few frequencies near the first natural frequency of the structure, can detect local damage accurately and the latter exhibits better performance than the former in both numerical simulations and experiment.

Yin and Tang [56] extend the application of the indirect method to a cable-stayed bridge. They seek to identify cable tension loss and deck damage using the displacement response of a moving vehicle crossing over the bridge. The vehicle is modelled as a sprung mass and the VBI is simulated by a finite-element method. The approach is based on Proper Orthogonal Decomposition (POD) of the difference between the displacement responses of a vehicle passing the damaged 
and the healthy bridges, respectively; this difference being considered as a relative displacement response. The method appears to perform quite well but has some drawbacks. The authors do not consider any road profile in their investigation which has been shown to have a very important influence on the vehicle response in previous studies. In addition, the authors recommend the use of a more complex VBI model. Finally, they note that highly sensitive equipment, such as laser displacement sensors, would be required due to the small amplitude of the displacement response to a single vehicle on a large bridge.

Miyamoto and Yabe [57, 58] develop a promising approach that could be termed crowd sourcing. They propose a bridge monitoring system based on vibration measurements on an in-service public bus. Safety, or damage indices are developed for short- and long-term monitoring, namely a structural anomaly parameter and a characteristic deflection, which are extracted from bus vibration measurements. In a field experiment, the effectiveness of using an accelerometer on the rear axle of the bus is compared with placing one at bridge mid-span and it is found that the approach is feasible as long as the same bus is used for all measurements. By taking a number of repeated measurements and averaging, the influence of noise is reduced. The characteristic deflection is estimated by using acceleration wave integrals obtained by Fourier transform and is considered to be relatively insensitive to vibration characteristics of the bridge and vehicle and dynamics related to road profile. The authors suggest that when the characteristic deflection has exceeded a certain limit, it can be judged that the bridge is showing signs of deterioration. Yabe et al. [59] extend the study of the monitoring system to include varying operating conditions such as weather, number of bus occupants, vehicle speed and oncoming traffic and illustrate its effectiveness.

$\mathrm{Li}$ and $\mathrm{Au}[60]$ suggest a multistage damage detection method based on modal strain energy and the genetic algorithm (GA). The modal strain energy based method estimates the damage location by calculating a damage indicator from the frequencies of the vehicle response for both the intact and damaged states of the bridge. Frequencies are extracted using Empirical Mode Decomposition. At the second stage, the identification problem is transformed into a global optimization problem and is solved by GA techniques. The approach can successfully determine the location of damage in a two-span continuous bridge with one damaged element. As in other studies, it is found that the method is influenced by the road profile and measurement noise. The authors compare the proposed method with wavelet-based and frequency-based damage detection methods in [61] to show its ability in the presence of a road profile.

Kong et al. [19] propose an indirect method for bridge damage detection utilizing one or more vehicles passing over the bridge. The concept of transmissibility is applied to the dynamic response of moving vehicles in a coupled vehicle-bridge interaction system. Acceleration responses are measured on two vehicles as they pass over the bridge. However, these vehicles are required to stop at different locations on the bridge for measurement. The authors extract the natural frequencies and modal shape squares of the bridge for damage detection using the transmissibility of these vehicle responses. Two different configurations are tested; firstly, one moving and one reference vehicle and secondly, two moving vehicles with constant spacing. It is found that vehicle transmissibility is sensitive to low-frequency bridge responses. The authors suggest that random traffic flows and vehicle speeds between $10 \mathrm{~m} / \mathrm{s}$ and $20 \mathrm{~m} / \mathrm{s}(36 \mathrm{~km} / \mathrm{h}$ and $72 \mathrm{~km} / \mathrm{h}$ ) may provide more suitable conditions for damage detection in the real world application of this method, although it is quite sensitive to road profile.

Cerda et al. [62] compare the results of an indirect bridge health monitoring technique with the direct approach in a laboratory scale model experiment in which bridge frequency changes are detected. In the experiment, a two-axle vehicle travels across a simply supported bridge consisting of an aluminium plate and angles. Changes to the bridge condition are made by adding localized mass at mid-span and bridge frequency changes are identified by averaging the short-time Fourier transform of acceleration measurements. Direct on-bridge measurements are found to be most stable in identifying frequency changes while the vehicle's front sprung mass measurement provided the best results for the indirect approach. The authors also note that lower vehicle speeds provide better results. Cerda et al. [63] extend the experimental investigation of the indirect bridge health monitoring technique to include two further damage scenarios and a greater number of data samples while new frequencybased damage features are used to identify the severity and location of damage. The two damage scenarios involve rotational restraint of a support and an increase of damping at different locations using adjustable dampers. To classify the damage features, a support vector machine classifier is used. The authors note that overall, damage of greater severity is detected with higher classification accuracy and also, damage detection is not very sensitive to vehicle speed. However, it is also acknowledged that the technique requires training data. Lederman et al. [64] expand on the work of Cerda et al. [63] by performing a regression on a large dataset of damage locations and severities, using the same experimental model. The authors demonstrate that the new method can provide better resolution in terms of damage location and severity. Chen et al. [65] suggest the application of the concept of classification to indirect bridge structural health monitoring. Generally, classification is a signal processing approach whose purpose is to design a map that relates each input with a predefined class label. Although the authors are aiming to improve the concept of classification, the proposed method has been applied well in indirect approaches.

Tsai et al. [66] investigate a railway track inspection method but also study the possibility of detecting the response of the bridge in that of the inspection car. However, it is found that bridge responses and frequencies could not be easily identified by the inspection car without a sophisticated analysis while the duration of the vehicle crossing is also identified as being a drawback of such an approach. This highlights some practical considerations for real-world applications in highway and potential railway applications. 


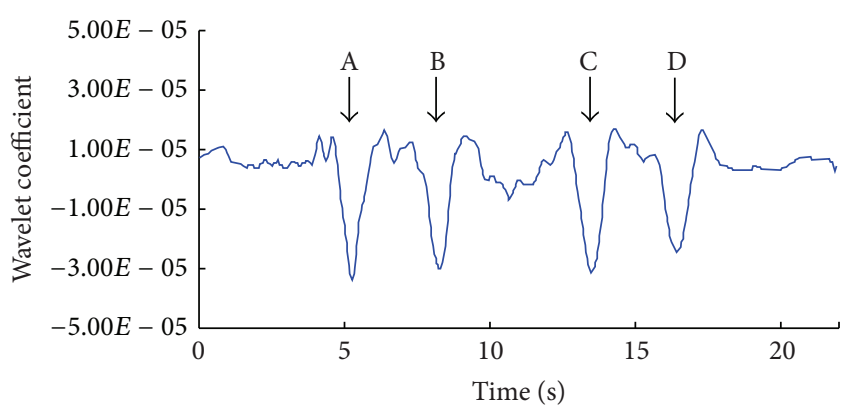

FIGURE 9: Wavelet transform of vehicle displacement when two cracks are considered at $L / 3$ and $2 L / 3$ of the beam with $30 \%$ crack depth and speed of $2 \mathrm{~m} / \mathrm{s}$. (A: first axle passing $L / 3$, B: second axle passing $L / 3$, C: first axle passing $2 L / 3$, and $\mathrm{D}$ : second axle passing $2 L / 3)$, after [17].

4.2. Wavelet Transform. A number of damage detection approaches incorporating wavelet theory have been proposed. Nguyen and Tran [17] apply a Symlet wavelet transform to the displacement response of a moving vehicle to identify the existence and location of cracks in a bridge. Theoretical simulations are carried out using a cracked finite element (FE) beam model and a 4 degree-of-freedom halfcar vehicle model. A two-crack scenario is investigated for varying vehicle speed $(2 \mathrm{~m} / \mathrm{s}-40 \mathrm{~m} / \mathrm{s})$, while crack depth is also varied as a percentage of the beam depth. Peaks at particular scales are observed in the wavelet transform of the vehicle displacement response when it passes over crack locations while crack depths of up to $10 \%$ are detected (Figure 9). It is found that deeper cracks are easier to detect while higher speeds provide poorer detection ability. The effect of white noise on crack detection is investigated and for $6 \%$ noise, a $50 \%$ crack depth is detected at $2 \mathrm{~m} / \mathrm{s}$. Overall, low speeds are recommended for accurate crack detection using this approach, which would have implications in practice, similar to a number of other indirect approaches presented in previous sections. Experimental testing is recommended by the authors who do not consider the road profile in their study.

Khorram et al. [18] also carry out a numerical investigation to compare the performance of two wavelet-based damage detection approaches. A very simple VBI model is used to compare two methods which utilize a Gaussian 4 wavelet transform: a "fixed sensor approach" and a "moving sensor approach," which are direct and indirect methods, respectively. The continuous wavelet transform (CWT) coefficients of time varying beam and vehicle displacements, respectively, are used to identify cracks which are modelled as rotational springs connecting elements. The vehicle or "moving" sensor is found to be more effective than the fixed sensor and small cracks with a depth of more than $10 \%$ of beam depth are detected. The authors develop a damage index which has an explicit expression and can identify crack depth as well as location. Although the proposed damage index shows good performance, the vehicle is idealized as a moving force and therefore does not consider the interaction between the vehicle and bridge or the effect of road profile.
McGetrick and Kim $[67,68]$ apply a CWT to the dynamic response of a vehicle passing over a bridge. It is shown that when the axle passes over a damaged section, any discontinuity in the signal affects the CWT coefficients, allowing damage to be identified and located. Based on these coefficients, a damage indicator (DI) is formulated which can distinguish between different damage levels, regardless of vehicle speed. Theoretical, experimental and field investigations are performed showing that the resulting DIs for the bridge and vehicle follow similar patterns. In simulations, vehicle speeds of 2, 5, 10, 15 and $20 \mathrm{~m} / \mathrm{s}$ are tested. Lower vehicle speeds provide higher resolution allowing damage detection to be located more accurately. However, it is difficult to distinguish between different artificial damage scenarios in the field experiment for the test vehicle speed of $40 \mathrm{~km} / \mathrm{h}$ $(11.1 \mathrm{~m} / \mathrm{s})$. A pattern-adapted wavelet is formulated and found to be beneficial for damage localisation in theoretical and experimental investigations; however its formulation requires prior knowledge or accurate estimation of bridge damage discontinuities [69]. The vehicle's transverse position on the road is also highlighted as having a significant influence on the DI's sensitivity to damage level, which may have implications for such an approach in practice.

These findings suggest that there are several advantages supporting further investigation of the use of wavelets for indirect bridge damage detection. In indirect damage detection, the vehicle is only on the bridge for a short length of time. This creates a challenge for conventional signal processing techniques (such as FFT), that are designed for infinite time series. Wavelets do not have this limitation which makes them particularly suited to this particular problem.

Wavelet methods have demonstrated the potential to detect, quantify and locate bridge damage, that is, to achieve level 3 SHM [6, 7]. However, further investigation is recommended to improve damage detection accuracy at higher vehicle speeds. Currently, a drawback is the necessity to close bridge lanes due to low operational speeds of around $2 \mathrm{~m} / \mathrm{s}$ $(7.2 \mathrm{~km} / \mathrm{h})$ but there is a possibility that the advantages of a highly accurate wavelet-based method could offset this. In addition, unless the vehicle's transverse position on the bridge is well controlled, there is a need to overcome the high sensitivity to its variation.

4.3. Traffic Speed Deflectometer. The prototype rolling weight deflectometer (RWD), presented first by Briggs et al. [70] in the United States, was designed for the indirect measurement of road pavement stiffness. It is proposed as a replacement for the falling weight deflectometer (FWD) which determines stiffness but which must stop at each test site for several minutes. More recently, the "High-speed Deflectograph," later renamed the "traffic speed deflectometer" (TSD) has emerged, capable of performing stiffness surveys at speeds of up to $80 \mathrm{~km} / \mathrm{h}$, avoiding traffic disruption and expensive traffic management. The TSD is a collection of noncontact lasers mounted at equal spacing on a rigid beam, housed in the trailer of an articulated lorry. Laser vibrometers continuously and very accurately measure velocities (related to distances between sensors and the road surface). It is already a proven technology for flexible pavements and trials 
TABLE 1: Indirect bridge monitoring summary (SHM levels: 1 = detect existence of damage, $2=$ detect damage location, and $3=$ detect damage severity).

\begin{tabular}{|c|c|c|c|}
\hline Method & SHM level & Advantages & Drawbacks \\
\hline Natural frequency & 1 & $\begin{array}{l}\text { Simple. } \\
\text { Demonstrated in many } \\
\text { experimental works. Acceptable } \\
\text { vehicle speed. }\end{array}$ & $\begin{array}{l}\text { Not always sensitive to damage. } \\
\text { Low frequency resolution when } \\
\text { vehicle speed is high. }\end{array}$ \\
\hline
\end{tabular}

based methods

Damping $\quad 1 \quad$ Sensitive to damage. Complexity.

$\begin{array}{lll}\text { Mode shape } & 1 \text { and } 2 & \begin{array}{l}\text { Local information. } \\ \text { Sensitive to damage. }\end{array}\end{array}$

No experimental confirmation.

\begin{tabular}{|c|c|c|c|c|}
\hline & & & & No experimental confirmation. \\
\hline \multirow{4}{*}{$\begin{array}{l}\text { Nonmodal parameter } \\
\text { based methods }\end{array}$} & Crowd sourcing & 1 & $\begin{array}{l}\text { Field experiments: ongoing in } \\
\text { service conditions. }\end{array}$ & $\begin{array}{l}\text { Only feasible using same vehicle } \\
\text { for all measurements. } \\
\text { Damage sensitivity unconfirmed } \\
\text { experimentally. }\end{array}$ \\
\hline & Wavelet & 1,2 , and 3 & Algorithms widely available. & $\begin{array}{l}\text { Low vehicle speed. } \\
\text { Relies on local anomalies in the } \\
\text { signal. } \\
\text { Results can be compromised by } \\
\text { edge effects. }\end{array}$ \\
\hline & TSD & 1 & $\begin{array}{l}\text { Very high accuracy of } \\
\text { measurements. } \\
\text { High vehicle speed. }\end{array}$ & $\begin{array}{l}\text { Expensive equipment. } \\
\text { No experimental confirmation. }\end{array}$ \\
\hline & Other & 1,2 , and 3 & $\begin{array}{l}\text { Novel numerical algorithms for } \\
\text { damage detection }\end{array}$ & $\begin{array}{l}\text { Limited experimental } \\
\text { confirmation. }\end{array}$ \\
\hline
\end{tabular}

have been carried out in a number of countries (e.g., Flintsch et al. [71]). For pavement applications, it only measures the "trough" (local depression) under a heavy axle as an indicator of pavement stiffness.

In numerical vehicle-bridge interaction simulations, Keenahan and OBrien [72] investigate the use of the TSD in a drive-by bridge damage detection context. A TSD model with three displacement sensors is proposed for bridge damage detection, which removes the bounce motion of the vehicle and the road profile influence. Different levels of damage are considered, and the approach also looks at changes in the transverse position of the vehicle and the addition of noise. OBrien and Keenahan [73] propose an alternative use of TSD vehicle data. They show that such a vehicle can accurately detect the "apparent profile," that is, the road profile that would be consistent with the velocity measurements if no bridge was present. They go on to show that this apparent profile is quite sensitive to bridge damage.

\section{Conclusions and Recommendations}

The authors' opinions on indirect methods of monitoring are summarised in Table 1. Historically, natural frequency is perhaps the most popular indicator of bridge damage and it can be found by indirect methods. However, it is generally accepted that the frequency changes caused by damage tend to be small and may be masked by the changes caused by environmental and operational conditions. Furthermore, frequency changes alone are not sufficient to uniquely define the location of structural damage [8]. Furthermore, most of the studies considered assume constant speed of the passing vehicles while in practice; it may not be easy to maintain this during the vehicle crossing. This factor becomes more significant when the signals of two axles are subtracted to minimise the influence of road profile. Therefore, speed variation should be investigated in future studies. The bridge damping coefficient can also be found from the response to a passing vehicle and most of the influence of road profile can be removed by subtracting the signals from successive trailer axles, provided those axles have identical properties. It is reasonable to assume that cracking in concrete structures would increase damping but it is less clear if such a link exists for steel structures. Furthermore, it is widely reported that damping is strongly influenced by environmental factors such as temperature which is likely to mask the effects of damage.

Mode shapes and modal curvature are generally considered to be good indicators of bridge damage, especially local damage that may cause a spatial discontinuity in the response. However, finding mode shapes indirectly remains a challenge. Recently, identification of bridge mode shapes from the vehicle response has attracted considerable interest. Zhang et al. [14] model a tapping vehicle (with an oscillating mass on board) but, even at very slow speeds, only the first mode shape is found accurately and applying such a device in 
the case of a real bridge tends to be expensive. Other authors $[15,46,47]$ do not require a tapping vehicle but the accuracy is generally poor unless there is a little measurement noise and the road surface is perfectly smooth. However, indirect mode shape-based methods have good potential for further development in the future due to the strong influence of local damage on mode shape.

There have been several attempts to detect damage without finding the conventional dynamic properties of the bridge (e.g., Bu et al. [52]; Kim and Kawatani [53]; McGetrick [54]). Many of these authors identify evidence of damage in wavelet transforms of the measured signal. Wavelets are a means of amplifying particular features, such as discontinuities in a signal and do not need long time series for good results. However, there are edge effects near the beginning and end of a bridge which can cause problems in these areas.

A particularly promising development is the concept of using vehicle-mounted laser vibrometers to obtain highly accurate measurements of the relative velocity (and hence displacement) between a moving vehicle and a bridge. OBrien and Keenahan [73] have developed a damage indicator from such measurements that appears to be highly damagesensitive. There is scope for a great deal of further development using such vehicles as the accuracy of the measurement should allow relatively minor damage to be detected.

A major obstacle to any form of rapid damage detection is temperature/environment which can have a significant effect on most damage indicators and whose influence is difficult to distinguish from real damage. This can be addressed by instrumenting vehicles that travel on the same route frequently as this allows environmental effects to be averaged out.

In summary, the main challenges for indirect bridge monitoring methods are

(i) the road profile,

(ii) the limited VBI time,

(iii) environmental effects.

Good progress has been made in addressing the influence of the road profile with the concept of subtracting signals from identical axles, although this requires very high measurement accuracy. The speed of the vehicle means that it is only present on the bridge for a limited time. This results in an inevitable shortage of vehicle-bridge interaction measurement data. Some studies require that speeds are very slow to address this problem but this is not ideal on busy roads where congestion may result. The final challenge in indirect monitoring is interference from environmental effects such as temperature. The most promising approach to tackle this issue is indirect monitoring using vehicles that repeatedly pass over the bridge. The potential of indirect methods is well illustrated in the literature, as discussed in this paper. Therefore, overcoming these challenges would represent a big step towards successful implementation of indirect bridge monitoring methods in practice.

\section{Conflict of Interests}

The authors declare that there is no conflict of interests regarding the publication of this paper.

\section{Acknowledgment}

The authors wish to express their gratitude for the financial support received from the Irish Research Council's Ph.D. in Sustainable Development Graduate Research Education Programme.

\section{References}

[1] S. L. Davis and D. Goldberg, The Fix We're In For: The State of Our Nation's Bridges 2013, Transportation for America, Washington, DC, USA, 2013.

[2] A.Žnidarič, V. Pakrashi, E. O’Brien, and A. O’Connor, “A review of road structure data in six European countries," Proceedings of the ICE: Urban Design and Planning, vol. 164, no. 4, pp. 225-232, 2011.

[3] P. Chupanit and C. Phromsorn, "The importance of bridge health monitoring," International Science Index, vol. 6, pp. 135138, 2012.

[4] Y. Fujino and D. M. Siringoringo, "Bridge monitoring in Japan: the needs and strategies," Structure and Infrastructure Engineering, vol. 7, no. 7-8, pp. 597-611, 2011.

[5] F. Akgül, "A BMS development project with an integrated inspection program," in Bridge Maintenance, Safety, Management, Resilience and Sustainability, chapter 360, pp. 2659-2665, CRC Press, New York, NY, USA, 2012.

[6] A. Rytter, Vibration Based Inspection of Civil Engineering Structures, Aalborg University, Aalborg, Denmark, 1993.

[7] E. P. Carden and P. Fanning, "Vibration based condition monitoring: a review," Structural Health Monitoring, vol. 3, no. 4, pp. 355-377, 2004.

[8] W. Fan and P. Z. Qiao, "Vibration-based damage identification methods: a review and comparative study," Structural Health Monitoring, vol. 10, no. 1, pp. 83-111, 2011.

[9] H. C. Gomez, P. J. Fanning, M. Q. Feng, and S. Lee, "Testing and long-term monitoring of a curved concrete box girder bridge," Engineering Structures, vol. 33, no. 10, pp. 2861-2869, 2011.

[10] Y.-B. Yang, C. W. Lin, and J. D. Yau, "Extracting bridge frequencies from the dynamic response of a passing vehicle," Journal of Sound and Vibration, vol. 272, no. 3-5, pp. 471-493, 2004.

[11] Y. B. Yang and C. W. Lin, "Vehicle-bridge interaction dynamics and potential applications," Journal of Sound and Vibration, vol. 284, no. 1-2, pp. 205-226, 2005.

[12] C. W. Lin and Y. B. Yang, "Use of a passing vehicle to scan the fundamental bridge frequencies: an experimental verification," Engineering Structures, vol. 27, no. 13, pp. 1865-1878, 2005.

[13] P. J. McGetrick, A. González, and E. J. OBrien, "Theoretical investigation of the use of a moving vehicle to identify bridge dynamic parameters," Insight, vol. 51, no. 8, pp. 433-438, 2009.

[14] Y. Zhang, L. Q. Wang, and Z. H. Xiang, "Damage detection by mode shape squares extracted from a passing vehicle," Journal of Sound and Vibration, vol. 331, no. 2, pp. 291-307, 2012.

[15] Y. B. Yang, Y. C. Li, and K. C. Chang, "Constructing the mode shapes of a bridge from a passing vehicle: a theoretical study," Smart Structures and Systems, vol. 13, no. 5, pp. 797-819, 2014. 
[16] J. Keenahan, P. McGetrick, E. J. O'Brien, and A. González, "Using instrumented vehicles to detect damage in bridges," in Proceedings of the 15th International Conference on Experimental Mechanics, Porto, Portugal, 2012.

[17] K. V. Nguyen and H. T. Tran, "Multi-cracks detection of a beam-like structure based on the on-vehicle vibration signal and wavelet analysis," Journal of Sound and Vibration, vol. 329, no. 21, pp. 4455-4465, 2010.

[18] A. Khorram, F. Bakhtiari-Nejad, and M. Rezaeian, "Comparison studies between two wavelet based crack detection methods of a beam subjected to a moving load," International Journal of Engineering Science, vol. 51, pp. 204-215, 2012.

[19] X. Kong, C. S. Cai, and B. Kong, "Damage detection based on transmissibility of a vehicle and bridge coupled system," Journal of Engineering Mechanics, vol. 141, no. 1, Article ID 04014102, 2015.

[20] A. González, "Vehicle-bridge dynamic interaction using finite element modelling," in Finite Element Analysis, pp. 637-662, Sciyo, Rijeka, Croatia, 2010.

[21] A. González, E. J. OBrien, and P. J. McGetrick, "Detection of bridge dynamic parameters using an instrumented vehicle," in Proceedings of the 5th World Conference on Structural Control and Monitoring, Tokyo, Japan, 2010.

[22] A. González, E. J. OBrien, and P. J. McGetrick, "Identification of damping in a bridge using a moving instrumented vehicle," Journal of Sound and Vibration, vol. 331, no. 18, pp. 4115-4131, 2012.

[23] J. Keenahan, E. J. OBrien, P. J. McGetrick, and A. Gonzalez, "The use of a dynamic truck-trailer drive-by system to monitor bridge damping," Structural Health Monitoring, vol. 13, no. 2, pp. 143-157, 2014.

[24] Y. Oshima, Y. Kobayashi, T. Yamaguchi, and K. Sugiura, "Eigenfrequency estimation for bridges using the response of a passing vehicle with excitation system," in Proceedings of the 4th International Conference on Bridge Maintenance (IABMAS '08), 3037, p. 3030, Seoul, Republic of Korea, July 2008.

[25] Y. Oshima, K. Yamamoto, K. Sugiura, and T. Yamaguchi, "Estimation of bridge eigenfrequencies based on vehicle responses using ICA," in Proceedings of the 10th International Conference on Structural Safety and Reliability (ICOSSAR '09), Osaka, Japan, September 2009.

[26] Y. B. Yang and K. C. Chang, "Extracting the bridge frequencies indirectly from a passing vehicle: parametric study," Engineering Structures, vol. 31, no. 10, pp. 2448-2459, 2009.

[27] Y. B. Yang and K. C. Chang, "Extraction of bridge frequencies from the dynamic response of a passing vehicle enhanced by the EMD technique," Journal of Sound and Vibration, vol. 322, no. 4-5, pp. 718-739, 2009.

[28] C. W. Kim, R. Isemoto, T. Toshinami, M. Kawatani, P. J. McGetrick, and E. J. O’Brien, "Experimental investigation of drive-by bridge inspection," in Proceedings of the 5th International Conference on Structural Health Monitoring of Intelligent Infrastructure (SHMII-5 '11), Cancun, Mexico, December 2011.

[29] T. Toshinami, M. Kawatani, and C. W. Kim, "Feasibility investigation for identifying bridge's fundamental frequencies from vehicle vibrations," in Proceedings of the 5th International Conference on Bridge Maintenance, Safety and Management (IABMAS '10), pp. 317-322, Philadelphia, Pa, USA, July 2010.

[30] D. M. Siringoringo and Y. Fujino, "Estimating bridge fundamental frequency from vibration response of instrumented passing vehicle: analytical and experimental study," Advances in Structural Engineering, vol. 15, no. 3, pp. 417-433, 2012.
[31] Y. Fujino, K. Kitagawa, T. Furukawa, and H. Ishii, "Development of vehicle intelligent monitoring system (VIMS)," in Smart Structures and Materials 2005: Sensors and Smart Structures Technologies for Civil, Mechanical, and Aerospace Systems, vol. 5765 of Proceedings of SPIE, pp. 148-157, March 2005.

[32] Y. B. Yang, K. C. Chang, and Y. C. Li, "Filtering techniques for extracting bridge frequencies from a test vehicle moving over the bridge," Engineering Structures, vol. 48, pp. 353-362, 2013.

[33] W.-M. Li, Z.-H. Jiang, T.-L. Wang, and H.-P. Zhu, “Optimization method based on Generalized Pattern Search Algorithm to identify bridge parameters indirectly by a passing vehicle," Journal of Sound and Vibration, vol. 333, no. 2, pp. 364-380, 2014.

[34] A. Malekjafarian and E. J. OBrien, "Application of outputonly modal method to the monitoring of bridges using an instrumented vehicle," in Civil Engineering Research in Ireland, S. Nanukuttan and J. Goggins, Eds., Belfast, Belfast, Northern Ireland, 2014.

[35] Y. B. Yang, M. C. Cheng, and K. C. Chang, "Frequency variation in vehicle-bridge interaction systems," International Journal of Structural Stability and Dynamics, vol. 13, no. 2, Article ID 1350019, 2013.

[36] K. C. Chang and C. W. Kim, "Variability in bridge frequency induced by a parked vehicle," in Proceedings of the 4th KKCNN Symposium on Civil Engineering, pp. 75-79, Hyogo, Japan, 2011.

[37] Y.-B. Yang, W.-F. Chen, H.-W. Yu, and C. S. Chan, "Experimental study of a hand-drawn cart for measuring the bridge frequencies," Engineering Structures, vol. 57, pp. 222-231, 2013.

[38] R. O. Curadelli, J. D. Riera, D. Ambrosini, and M. G. Amani, "Damage detection by means of structural damping identification," Engineering Structures, vol. 30, no. 12, pp. 3497-3504, 2008.

[39] C. Modena, D. Sonda, and D. Zonta, "Damage localization in reinforced concrete structures by using damping measurements," Key Engineering Materials, vol. 167, no. 1, pp. 132-141, 1999.

[40] P. McGetrick, C. W. Kim, and E. J. OBrien, "Experimental investigation of the detection of bridge dynamic parameters using a moving vehicle," in Proceedings of the 23rd KKCNN Symposium on Civil Engineering, Taipei, Taiwan, 2010.

[41] C. W. Kim, R. Isemoto, P. J. Mcgetrick, M. Kawatani, and E. J. Obrien, "Drive-by bridge inspection from three different approaches," Smart Structures and Systems, vol. 13, no. 5, pp. 775-796, 2014.

[42] C. Williams and O. S. Salawu, "Damping as a damage indication parameter," in Proceedings of the 15th International Modal Analysis Conference (IMAC '97), pp. 1531-1536, Orlando, Fla, USA, February 1997.

[43] X. Q. Zhu and S. S. Law, "Wavelet-based crack identification of bridge beam from operational deflection time history," International Journal of Solids and Structures, vol. 43, no. 7-8, pp. 2299-2317, 2006.

[44] A. K. Pandey, M. Biswas, and M. M. Samman, "Damage detection from changes in curvature mode shapes," Journal of Sound and Vibration, vol. 145, no. 2, pp. 321-332, 1991.

[45] V. Arora, S. P. Singh, and T. K. Kundra, "Damped model updating using complex updating parameters," Journal of Sound and Vibration, vol. 320, no. 1-2, pp. 438-451, 2009.

[46] Y. Oshima, K. Yamamoto, and K. Sugiura, "Damage assessment of a bridge based on mode shapes estimated by responses of passing vehicles," Smart Structures and Systems, vol. 13, no. 5, pp. 731-753, 2014. 
[47] A. Malekjafarian and E. J. OBrien, "Identification of bridge mode shapes using short time frequency domain decomposition of the responses measured in a passing vehicle," Engineering Structures, vol. 81, pp. 386-397, 2014.

[48] Y. B. Yang, Y. C. Li, and K. C. Chang, "Effect of road surface roughness on the response of a moving vehicle for identification of bridge frequencies," Interaction and Multiscale Mechanics, vol. 5, no. 4, pp. 347-368, 2012.

[49] Y. B. Yang, Y. C. Lee, and K. C. Chang, "Effect of road surface roughness on extraction of bridge frequencies by moving vehicle," in Mechanics and Model-Based Control of Advanced Engineering Systems, pp. 295-305, Springer, Vienna, Austria, 2014.

[50] Y. B. Yang, Y. C. Li, and K. C. Chang, "Using two connected vehicles to measure the frequencies of bridges with rough surface: a theoretical study," Acta Mechanica, vol. 223, no. 8, pp. 1851-1861, 2012.

[51] A. González, E. J. O’Brien, Y.-Y. Li, and K. Cashell, “The use of vehicle acceleration measurements to estimate road roughness," Vehicle System Dynamics, vol. 46, no. 6, pp. 483-499, 2008.

[52] J. Q. Bu, S. S. Law, and X. Q. Zhu, "Innovative bridge condition assessment from dynamic response of a passing vehicle," Journal of Engineering Mechanics, vol. 132, no. 12, pp. 1372-1379, 2006.

[53] C. W. Kim and M. Kawatani, "Challenge for a drive-by bridge inspection," in Proceedings of the 10th International Conference on Structural Safety and Reliability (ICOSSAR '09), pp. 758-765, Osaka, Japan, 2009.

[54] P. J. McGetrick, The use of an instrumented vehicle to monitor transport infrastructure [Ph.D. thesis], University College Dublin, Dublin, Ireland, 2012.

[55] Y. Zhang, S. T. Lie, and Z. H. Xiang, "Damage detection method based on operating deflection shape curvature extracted from dynamic response of a passing vehicle," Mechanical Systems and Signal Processing, vol. 35, no. 1-2, pp. 238-254, 2013.

[56] S.-H. Yin and C.-Y. Tang, "Identifying cable tension loss and deck damage in a cable-stayed bridge using a moving vehicle," Journal of Vibration and Acoustics, Transactions of the ASME, vol. 133, no. 2, Article ID 021007, 2011.

[57] A. Miyamoto and A. Yabe, "Bridge condition assessment based on vibration responses of passenger vehicle," Journal of Physics: Conference Series, vol. 305, no. 1, Article ID 012103, 2011.

[58] A. Miyamoto and A. Yabe, "Development of practical health monitoring system for short- and medium-span bridges based on vibration responses of city bus," Journal of Civil Structural Health Monitoring, vol. 2, no. 1, pp. 47-63, 2012.

[59] A. Yabe, A. Miyamoto, S. Isoda, and N. Tani, "Development of bridge monitoring system for short- and medium-span bridges based on bus vibration," Journal of Japan Society of Civil Engineers, Series F4 (Construction and Management), vol. 69, no. 2, pp. 102-120, 2013.

[60] Z. H. Li and F. T. K. Au, "Damage detection of a continuous bridge from response of a moving vehicle," Shock and Vibration, vol. 2014, Article ID 146802, 7 pages, 2014.

[61] Z. Li and F. T. K. Au, "Damage detection of bridges using response of vehicle considering road surface roughness," International Journal of Structural Stability and Dynamics, 2014.

[62] F. Cerda, J. Garrett, J. Bielak et al., "Indirect structural health monitoring in bridges: scale experiments," in Bridge Maintenance, Safety, Management, Resilience and Sustainability, pp. 346-353, 2012.
[63] F. Cerda, S. Chen, J. Bielak, J. H. Garrett, P. Rizzo, and J. KovaČević, "Indirect structural health monitoring of a simplified laboratory-scale bridge model," Smart Structures and Systems, vol. 13, no. 5, pp. 849-868, 2014.

[64] G. Lederman, Z. Wang, J. Bielak et al., "Damage quantification and localization algorithms for indirect SHM of bridges," in Bridge Maintenance, Safety, Management and Life Extension, chapter 83, pp. 640-647, CRC Press, New York, NY, USA, 2014.

[65] S. H. Chen, F. Cerda, P. Rizzo, J. Bielak, J. H. Garrett, and J. Kovacevic, "Semi-supervised multiresolution classification using adaptive graph filtering with application to indirect bridge structural health monitoring," IEEE Transactions on Signal Processing, vol. 62, pp. 2879-2893, 2014.

[66] H.-C. Tsai, C.-Y. Wang, N. E. Huang, T.-W. Kuo, and W.H. Chieng, "Railway track inspection based on the vibration response to a scheduled train and the Hilbert-Huang transform," Proceedings of the Institution of Mechanical Engineers, Part F: Journal of Rail and Rapid Transit, 2014.

[67] P. J. McGetrick and C. W. Kim, "A parametric study of a drive by bridge inspection system based on the Morlet wavelet," Key Engineering Materials, vol. 569-570, pp. 262-269, 2013.

[68] P. J. McGetrick and C. W. Kim, "An indirect bridge inspection method incorporating a wavelet-based damage indicator and pattern recognition," in Proceedings of the 9th International Conference on Structural Dynamics (EURODYN '14), Porto, Portugal, July 2014.

[69] P. McGetrick and C. A. Kim, "A wavelet based drive-by bridge inspection system," in Proceedings of the 7th International Conference on Bridge Maintenance Safety and Management (IABMAS '14), Shanghai, China, 2014.

[70] R. C. Briggs, R. F. Johnson, R. N. Stubstad, and L. Pierce, "A comparison of the rolling weight deflectometer with the falling weight deflectometer," American Society for Testing and Materials, vol. 1375, pp. 444-456, 2000.

[71] G. W. Flintsch, B. Ferne, B. Diefenderfer, S. Katicha, J. Bryce, and S. Nell, "Evaluation of Traffic Speed Continuous Deflection Devices," in Proceedings of the 91st Annual Meeting, Transport Research Board, Washington, DC, USA, January 2012.

[72] J. Keenahan and E. J. OBrien, "Allowing for a rocking datum in the analysis of drive-by bridge inspections," in Civil Engineering Research in Ireland, S. Nanukuttan and J. Goggins, Eds., pp. 117124, Belfast, UK, 2014.

[73] E. J. OBrien and J. Keenahan, "Drive-by damage detection in bridges using the apparent profile," Structural Control and Health Monitoring, 2014. 

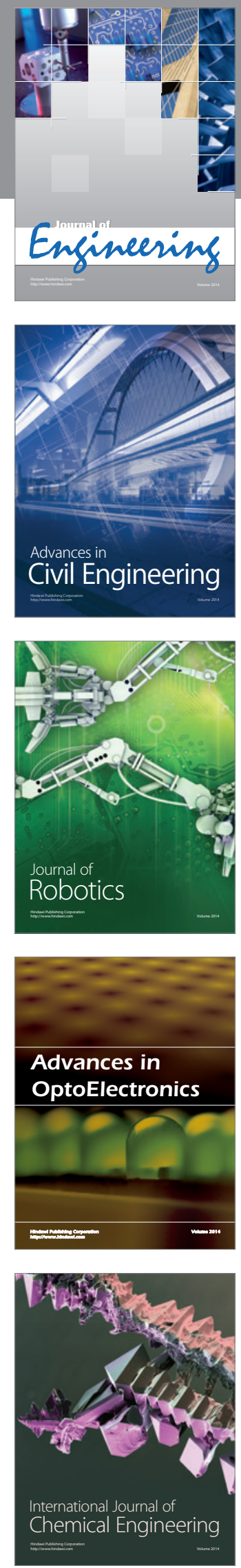

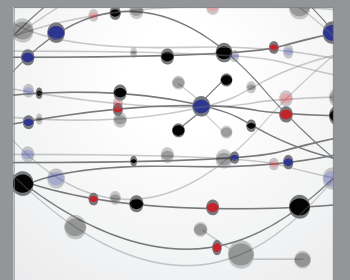

The Scientific World Journal
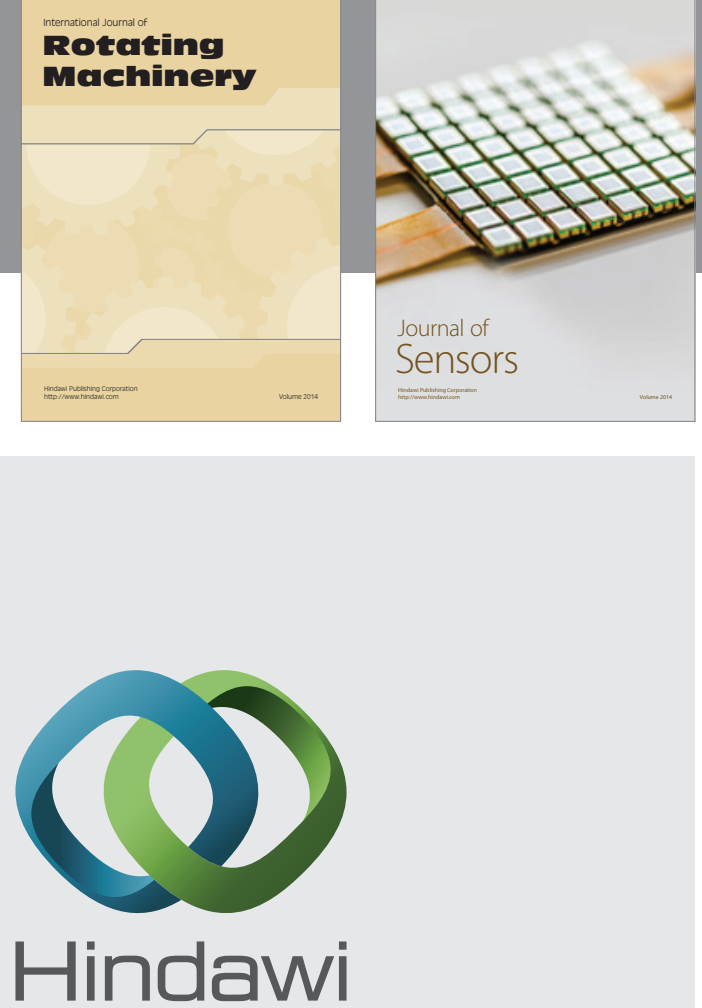

Submit your manuscripts at http://www.hindawi.com
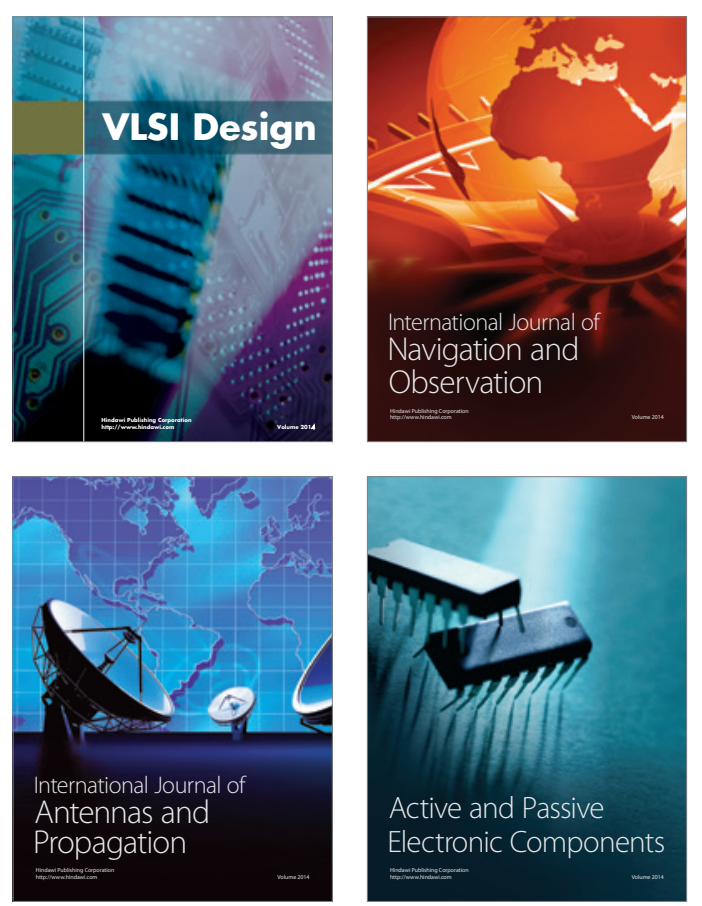
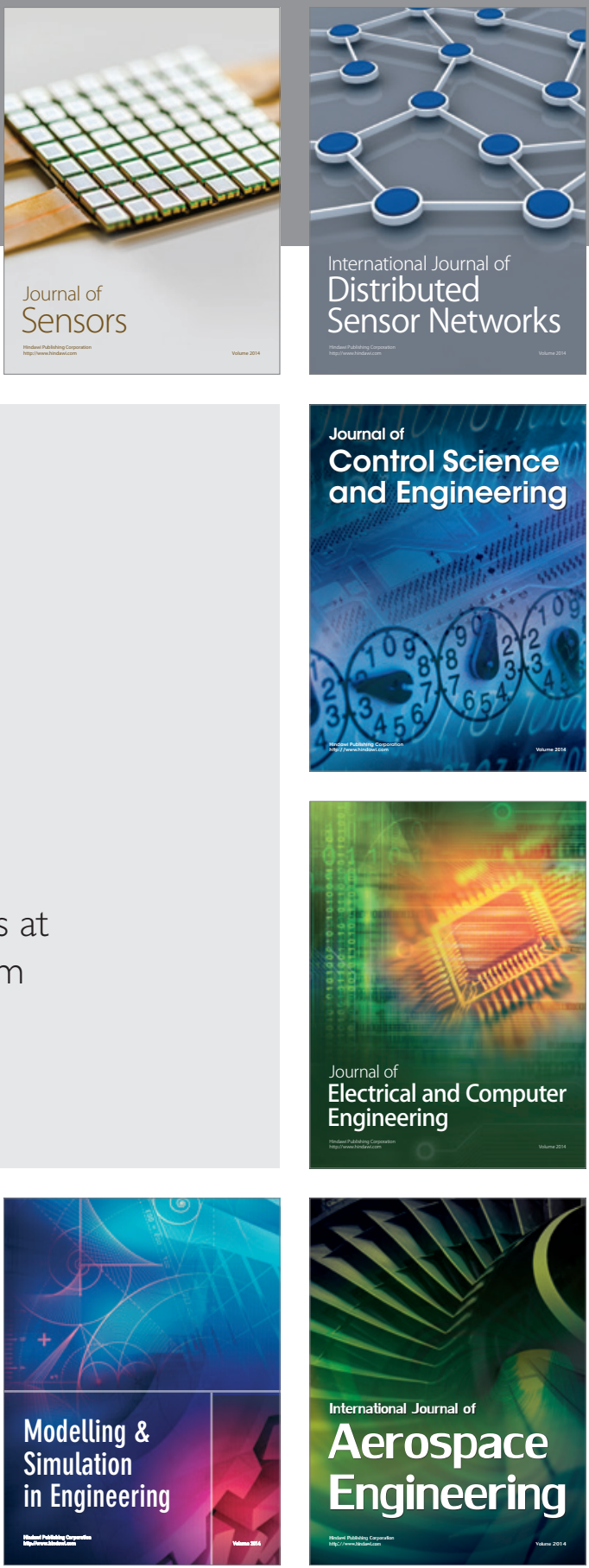

Journal of

Control Science

and Engineering
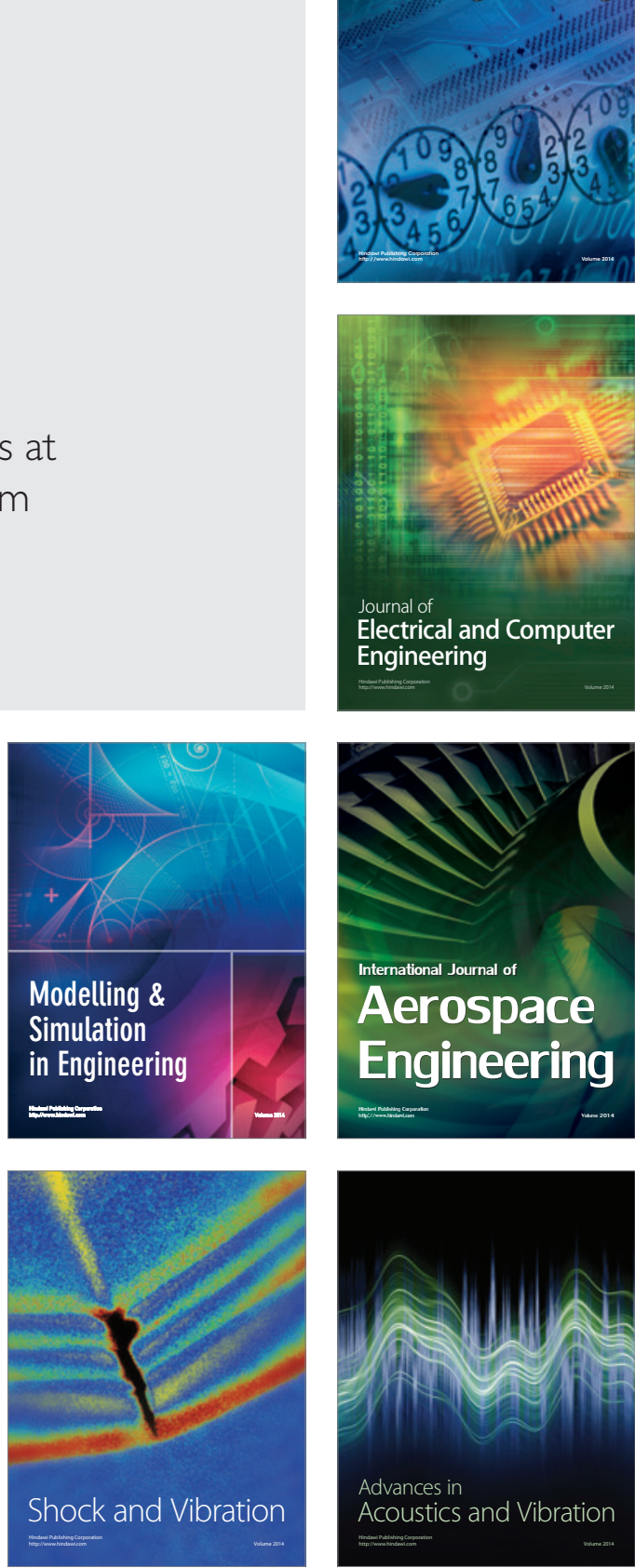